\title{
One-loop light-cone QCD, effective action for reggeized gluons and QCD RFT calculus
}

\author{
S. Bondarenko ${ }^{1, a}$, L. Lipatov ${ }^{2,3}$, S. Pozdnyakov ${ }^{1}$, A. Prygarin ${ }^{1}$ \\ ${ }^{1}$ Physics Department, Ariel University, 40700 Ariel, Israel \\ 2 St. Petersburg State University, St. Petersburg 199034, Russia \\ ${ }^{3}$ Petersburg Nuclear Physics Institute, Gatchina 188300, Russia
}

Received: 17 August 2017 / Accepted: 4 September 2017 / Published online: 21 September 2017

(C) The Author(s) 2017. This article is an open access publication

\begin{abstract}
The effective action for reggeized gluons is based on the gluodynamic Yang-Mills Lagrangian with external current for longitudinal gluons added, see Lipatov (Nucl Phys B 452:369, 1995; Phys Rep 286:131, 1997; Subnucl Ser 49:131, 2013; Int J Mod Phys Conf Ser 39:1560082, 2015; Int J Mod Phys A 31(28/29):1645011, 2016; EPJ Web Conf 125:01010, 2016). On the base of classical solutions, obtained in Bondarenko et al. (Eur Phys J C 77(8):527, 2017), the one-loop corrections to this effective action in light-cone gauge are calculated. The RFT calculus for reggeized gluons similarly to the RFT introduced in Gribov (Sov Phys JETP $26: 414,1968)$ is proposed and discussed. The correctness of the results is verified by calculation of the propagators of $A_{+}$and $A_{-}$reggeized gluons fields and application of the obtained results is discussed as well.
\end{abstract}

\section{Introduction}

The action for the interaction of reggeized gluons was introduced in the series of papers [1-6] and describes multi-Regge processes at high energies; see [9-22]. There are the following important applications of this action: it can be used for the calculation of production amplitudes in different scattering processes and calculation of sub-leading, unitarizing corrections to the amplitudes and production vertices; see [16,9-32]. The last task can be considered as a construction of the RFT (Regge Field Theory) based on the interaction of the fields of reggeized gluons, where different vertices of the interactions are introduced and calculated. The phenomenological RFT based on the Pomeron degrees of freedom was introduced in [8]. From this point of view we consider the effective action for reggeized gluons as RFT calculus based

\footnotetext{
a e-mail: sergeyb@ariel.ac.il
}

on the degrees of freedom expressed through colored reggeon fields (reggeized gluons).

The construction of an RFT based on a QCD Lagrangian requires the knowledge of solutions of classical equations of motion in terms of reggeon fields $A_{+}$and $A_{-}$. Inserting these solutions again in the Lagrangian we can develop field theory fully in terms of reggeon fields, with loop corrections to the action determined in terms of these fields as well. A subsequent expansion of the action in terms of the reggeon fields will produce all possible vertices of interactions of the fields, with a precision determined by the precision of calculations in the framework of the QFT. From the QFT point of view, therefore, the problem of interest is the calculation of the one-loop effective action for gluon QCD Lagrangian with added external current by use of the non-trivial classical solutions expressed in terms of new degrees of freedom; see [7]. These calculations of the one-loop corrections to the effective action we perform in light-cone gauge using classical solutions from [7]. The correctness of the obtained results can be checked by calculations of functions which are well known in the small-x BFKL approach [33-38]. The basic function is the gluon Regge trajectory, which determines the form of the propagator of the reggeized gluon fields $A_{+}$and $A_{-}$. This propagator in the proposed framework can be considered as an operator inverse to the effective vertex of interaction of reggeon fields, and this check is performed in the paper. There are also other possibilities to verify the self-consistency of the approach. For example, it might be a calculation of the BFKL kernel, which is an effective vertex of interactions of four reggeons, or a calculation of the triple Pomeron vertex, see [39-41], which is the interaction vertex of six reggeon fields. These calculations will be considered further in separate publications.

Thus, below, we calculate a one-loop effective action for reggeized gluons and calculate propagators for $A_{+}$and $A_{-}$ reggeon fields. Respectively, in Sect. 2, we recall the main 
results obtained for the classical solution of the effective action for reggeized gluons. In Sect. 3 we consider the expansion of the Lagrangian in terms of fluctuations around these classical solutions, whereas in Sect. 4 we calculate the oneloop correction to the classical action obtaining an effective one-loop action in terms of the reggeon fields. In Sect. 5 we discuss RFT calculus based on the effective action and in Sect. 6 we verify the correctness of the result calculating the propagator of the reggeized gluon fields. Section 7 is the conclusion of the paper, but we also have Appendices A, B, and $\mathrm{C}$ where the main calculations related to the result are presented.

\section{Effective action for reggeized gluons and classical equations of motion}

The effective action, see [1-6], is a non-linear gauge invariant action which correctly reproduces the production of the particles in direct channels at a quasi-multi-Regge kinematics. It is written for the interactions local in rapidity of the physical gluons in direct channels in some rapidity interval $(y-\eta / 2, y+\eta / 2)$. The interaction between the different clusters of gluons at different though very close rapidities can be described with the help of reggeized gluon fields ${ }^{1}$ $A_{-}$and $A_{+}$interacting in crossing channels. Those interactions are non-local in rapidity space. This non-local term is not included in the action, the term of interaction between the reggeon fields in the action is local in rapidity and can be considered a kind of renormalization term in the Lagrangian. The action is gauge invariant and is written in the covariant form in terms of the gluon field $v$ as

$$
\begin{aligned}
S_{\text {eff }}= & -\int \mathrm{d}^{4} x\left(\frac{1}{4} F_{\mu \nu}^{a} F_{a}^{\mu \nu}+\operatorname{tr}\left[v_{+} J^{+}\left(v_{+}\right)\right.\right. \\
& \left.\left.-A_{+} j_{\text {reg }}^{+}+v_{-} J^{-}\left(v_{-}\right)-A_{-} j_{\text {reg }}^{-}\right]\right),
\end{aligned}
$$

where

$J^{ \pm}\left(v_{ \pm}\right)=O\left(x^{ \pm}, v_{ \pm}\right) j_{\text {reg }}^{ \pm}$,

where the $O\left(x^{ \pm}, v_{ \pm}\right)$are operators, see [1-6], Appendix A and

$j_{\text {reg } a}^{ \pm}=\frac{1}{C(R)} \partial_{i}^{2} A_{a}^{ \pm}$

is a reggeon current, where $C(R)$ is the eigenvalue of a Casimir operator in the representation $\mathrm{R}$ with $C(R)=N$ in the case of the adjoint representation used in the paper. Further in the calculations we will use the form of the reggeon

\footnotetext{
1 We use the Kogut-Soper convention for the light cone for the lightcone definitions with $x_{ \pm}=\left(x_{0} \pm x_{3}\right) / \sqrt{2}$ and $x_{ \pm}=x^{\mp}$.
}

current Eq. (3) borrowed from the CGC (Color Glass Condensate) approach, see [42-53], where this current is written in terms of some color density function defined as

$\partial_{i} \partial_{-} \rho_{a}^{i}=-\frac{1}{N} \partial_{\perp}^{2} A_{a}^{+}$,

or

$\rho_{a}^{i}=\frac{1}{N} \partial_{-}^{-1}\left(\partial^{i} A_{-}^{a}\right) ;$

see [7] for details. There are additional kinematical constraints for the reggeon fields

$\partial_{-} A_{+}=\partial_{+} A_{-}=0$,

corresponding to a strong ordering of the Sudakov components in the multi-Regge kinematics; see [1-6]. Everywhere, as usual, $\partial_{i}$ denotes the derivative on transverse coordinates. Under variation of the gluon fields these currents reproduce Lipatov's induced currents

$\delta\left(v_{ \pm} J^{ \pm}\left(v_{ \pm}\right)\right)=\left(\delta v_{ \pm}\right) j_{\mp}^{\text {ind }}\left(v_{ \pm}\right)=\left(\delta v_{ \pm}\right) j^{ \pm}\left(v_{ \pm}\right)$,

with the notation $j_{\mp}^{\text {ind }}=j^{ \pm}$introduced. This current possesses a covariant conservation property:

$\left(D_{ \pm} j_{\mp}^{\text {ind }}\left(v_{ \pm}\right)\right)^{a}=\left(D_{ \pm} j^{ \pm}\left(v_{ \pm}\right)\right)^{a}=0$.

Here and further we denote the induced current in the component form in the adjoint representation ${ }^{2}$

$$
\begin{aligned}
j_{a}^{ \pm}\left(v_{ \pm}\right) & =-l \operatorname{tr}\left[T_{a} j^{ \pm}\left(v_{ \pm}\right)\right]=\frac{1}{N} \operatorname{tr}\left[f_{a} O f_{b} O^{T}\right]\left(\partial_{i}^{2} A_{\mp}^{b}\right) \\
& =\frac{1}{N} U^{a b}\left(\partial_{i}^{2} A_{\mp}^{b}\right) ;
\end{aligned}
$$

see [7] and Appendix A as well. It was shown in [7] that if the LO value of the classical gluon field in the solutions of the equations of motion is fixed as

$v_{ \pm}=A_{ \pm}$

and if the self-consistency of the solutions is required, the currents of the Lagrangian in Eq. (1) are reproduced directly in the form of Eq. (2) without any additional conditions. Now, applying the light-cone gauge $v_{-}=0$, the equations of motion can be solved; see the form of the classical solutions in [7]. Therefore, the general expressions for the gluon fields can be written in the following form:

$v_{i}^{a} \rightarrow v_{i c l}^{a}+\varepsilon_{i}^{a}, v_{+}^{a} \rightarrow v_{+c l}^{a}+\varepsilon_{+}^{a}$,

where the integration on fluctuations around the classical solutions provides loop corrections to the "net" contribution which is based on the classical solutions only.

\footnotetext{
${ }^{2}$ We use the definition $\left(T_{a}\right)_{b c}=-l f_{a b c}$ of the matrices and write only "external" indices of the $f_{a b c}=\left(f_{a}\right)_{b c}$ matrix in the trace.
} 


\section{Expansion of the Lagrangian around the classical solution}

In this section we consider the first step in construction of the effective action of the approach, expanding the Lagrangian of Eq. (1) in terms of the fluctuations and classical fields. Inserting Eq. (11) in the Lagrangian the only corrections to orders $g^{2}$ and $\varepsilon^{2}$ will be preserved. This precision provides a one-loop correction to the "net" effective action; contributions from higher order loops will be considered in a separate publication.

The Lagrangian in light-cone gauge has the following form:

$L=-\frac{1}{4} F_{i j}^{a} F_{i j}^{a}+F_{i+}^{a} F_{i-}^{a}+\frac{1}{2} F_{+-}^{a} F_{+-}^{a}$,

where $F_{+-}^{a} F_{+-}^{a}$ term does not consist of transverse fluctuations and the $F_{i j}^{a} F_{i j}^{a}$ term does not consist of longitudinal fluctuations.

\subsection{The $F_{i+}^{a} F_{i-}^{a}$ term}

Inserting Eq. (11) in this term we obtain

$$
\begin{aligned}
F_{i+}^{a} \rightarrow & F_{i+}^{a}\left(v_{+}^{c l}, v_{i}^{c l}\right)+\left(D_{i}\left(v_{i}^{c l}\right) \varepsilon_{+}\right)^{a} \\
& -\left(D_{+}\left(v_{+}^{c l}\right) \varepsilon_{i}\right)^{a}+g f_{a b c} \varepsilon_{i}^{b} \varepsilon_{+}^{c}
\end{aligned}
$$

and

$F_{i-}^{a} \rightarrow F_{i-}^{a}\left(v_{i}^{c l}\right)-\partial_{-} \varepsilon_{i}^{a}$.

Therefore we have

$$
\begin{aligned}
F_{i+}^{a} F_{i-}^{a}= & \left(F_{i+}^{a} F_{i-}^{a}\right)_{c l}+F_{i-}^{a}\left(v_{i}^{c l}\right)\left(\left(D_{i}\left(v_{i}^{c l}\right) \varepsilon_{+}\right)^{a}\right. \\
& \left.-\left(D_{+}\left(v_{+}^{c l}\right) \varepsilon_{i}\right)^{a}\right)-g f_{a b c}\left(\partial_{-} v_{i}^{a c l}\right) \varepsilon_{i}^{b} \varepsilon_{+}^{c} \\
& -\left(\partial_{-} \varepsilon_{i}^{a}\right) F_{i+}^{a}\left(v_{+}^{c l}, v_{i}^{c l}\right)-\left(\partial_{-} \varepsilon_{i}^{a}\right)\left(D_{i}\left(v_{i}^{c l}\right) \varepsilon_{+}\right)^{a} \\
& +\left(\partial_{-} \varepsilon_{i}^{a}\right)\left(D_{+}\left(v_{+}^{c l}\right) \varepsilon_{i}\right)^{a}-g f_{a b c}\left(\partial_{-} \varepsilon_{i}^{a}\right) \varepsilon_{i}^{b} \varepsilon_{+}^{c} .
\end{aligned}
$$

In this expression we do not account for the term which is cubic in the fluctuations, the linear terms for the fluctuations are canceled because of the equations of motion. The terms for the quadratic to transverse fluctuations contribute to the corresponding propagator in the Lagrangian and the term which is quadratic with respect to the combination of transverse and longitudinal fluctuations we write as

$$
-g f_{a b c}\left(\partial_{-} v_{i}^{a c l}\right) \varepsilon_{i}^{b} \varepsilon_{+}^{c}-\left(\partial_{-} \varepsilon_{i}^{a}\right)\left(D_{i}\left(v_{i}^{c l}\right) \varepsilon_{+}\right)^{a}=J_{i}^{a} \varepsilon_{i}^{a} .
$$

Here the current

$$
J_{i}^{a}=\left(\varepsilon_{+}^{a} \partial_{-} \partial_{i}+g f_{a b c} \varepsilon_{+}^{c}\left(\partial_{-} v_{i}^{b c l}-v_{i}^{b c l} \partial_{-}\right)\right)
$$

is some effective current in the Lagrangian.
3.2 The $F_{i j}^{a} F_{i j}^{a}$ term

We have for the term $F_{i j}^{a} F_{i j}^{a}$

$F_{i j}^{a}=F_{i j}^{a}\left(v_{i}^{c l}\right)+\left(D_{i} \varepsilon_{j}\right)^{a}-\left(D_{j} \varepsilon_{i}\right)^{a}+g f_{a b c} \varepsilon_{i}^{b} \varepsilon_{j}^{c}$.

Therefore, accounting for the contributions which are only quadratic for the fluctuations, we obtain

$$
\begin{aligned}
-\frac{1}{4} F_{i j}^{a} F_{i j}^{a}= & -\frac{1}{4}\left(F_{i j}^{a} F_{i j}^{a}\right)_{c l}-\frac{1}{2} F_{i j}^{a}\left(v_{i}^{c l}\right)\left(\left(D_{i} \varepsilon_{j}\right)^{a}\right. \\
& \left.-\left(D_{j} \varepsilon_{i}\right)^{a}\right)-\frac{g}{2} F_{i j}^{a}\left(v_{i}^{c l}\right) f_{a b c} \varepsilon_{i}^{b} \varepsilon_{j}^{c} \\
& -\frac{1}{2}\left(D_{i} \varepsilon_{j}\right)^{a}\left(D_{i} \varepsilon_{j}\right)^{a}+\frac{1}{2}\left(D_{i} \varepsilon_{j}\right)^{a}\left(D_{j} \varepsilon_{i}\right)^{a},
\end{aligned}
$$

where as usual the linear terms for the fluctuations do not contribute to the effective action.

\subsection{The $F_{+-}^{a} F_{+-}^{a}$ term}

The term $F_{+-}^{a} F_{+-}^{a}$ consists of contributions from only longitudinal fluctuations. We have

$$
\begin{aligned}
\frac{1}{2} F_{+-}^{a} F_{+-}^{a} & \rightarrow \frac{1}{2}\left(\partial_{-} v_{+}^{a}\right)\left(\partial_{-} v_{+}^{a}\right)=-\frac{1}{2} v_{+}^{a c l}\left(\partial_{-}^{2} v_{+}^{a c l}\right) \\
& -\left(\partial_{-}^{2} v_{+}^{a c l}\right) \varepsilon_{+}^{a}-\frac{1}{2} \varepsilon_{+}^{a}\left(\partial_{-}^{2} \varepsilon_{+}^{a}\right) .
\end{aligned}
$$

The linear term in Eq. (20) is canceled due to the equation of motion, therefore only the first and third terms are considered further.

\subsection{The current term}

For the effective current term, taking into account that the linear term for the fluctuations is canceled due the equations of motion, we obtain with the required precision the following expansion in terms of longitudinal fluctuations:

$v_{+}^{a} J_{a}^{+}\left(v_{+}\right)=v_{+}^{a c l} J_{a c l}^{+}\left(v_{+}^{c l}\right)+\frac{1}{2}\left(\frac{\delta^{2}\left(v_{+}^{a} J_{a}^{+}\right)}{\delta v_{+}^{b} \delta v_{+}^{c}}\right)_{v_{+}=v_{+}^{c l}}^{x y} \varepsilon_{+x}^{b} \varepsilon_{+y}^{c}$.

We can write the same current's term as

$$
\begin{aligned}
v_{+}^{a} J_{a}^{+}\left(v_{+}\right)= & -v_{+}^{a c l} O^{a b}\left(v_{+}^{c l}\right)\left(\partial_{i} \partial_{-} \rho_{b}^{i}\right) \\
& -\frac{1}{2}\left(\frac{\delta U^{b a}\left(v_{+}\right)}{\delta v_{+}^{c}}\right)_{v_{+}=v_{+}^{c l}}^{x y}\left(\partial_{i} \partial_{-} \rho_{a}^{i}\right)_{x} \varepsilon_{+x}^{b} \varepsilon_{+y}^{c} .
\end{aligned}
$$

In order to calculate this expression we have to know the expansion of the following function:

$U^{a b}\left(v_{+}\right)=\operatorname{tr}\left[f_{a} O\left(v_{+}\right) f_{b} O^{T}\left(v_{+}\right)\right]$ 
with respect to the fluctuation of Eq. (11),

$v_{+}^{a} \rightarrow v_{+c l}^{a}+\varepsilon_{+}^{a}$.

Using Appendix A we have

$$
\begin{aligned}
U_{x}^{a b}\left(v_{+}\right)= & U_{x}^{a b}\left(v_{+0}^{c l}\right)+g\left(U_{1}^{a b}\right)_{x y}^{c} \varepsilon_{+y}^{c} \\
& +\frac{1}{2} g^{2}\left(U_{2}^{a b}\right)_{x y z}^{c d} \varepsilon_{+y}^{c} \varepsilon_{+z}^{d}+\cdots,
\end{aligned}
$$

where the integration on repeating $y, z$ indices is assumed. The coefficients of the expansion read

$$
\left(U_{1}^{a b}\right)_{x y}^{c}=\operatorname{tr}\left[f_{a} G_{x y}^{+} f_{c} O_{y} f_{b} O_{x}^{T}\right]+\operatorname{tr}\left[f_{c} G_{y x}^{+} f_{a} O_{x} f_{b} O_{y}^{T}\right]
$$

and

$$
\begin{aligned}
\left(U_{2}^{a b}\right)_{x y z}^{c d}= & \operatorname{tr}\left[f_{a} G_{x y}^{+} f_{c} G_{y z}^{+} f_{d} O_{z} f_{b} O_{x}^{T}\right] \\
& +\operatorname{tr}\left[f_{a} G_{x z}^{+} f_{d} G_{z y}^{+} f_{c} O_{y} f_{b} O_{x}^{T}\right] \\
& +\operatorname{tr}\left[f_{d} G_{z x}^{+} f_{a} G_{x y}^{+} f_{c} O_{y} f_{b} O_{z}^{T}\right] \\
& +\operatorname{tr}\left[f_{c} G_{y x}^{+} f_{a} G_{x z}^{+} f_{d} O_{z} f_{b} O_{y}^{T}\right] \\
& +\operatorname{tr}\left[f_{d} G_{z y}^{+} f_{c} G_{y x}^{+} f_{a} O_{x} f_{b} O_{z}^{T}\right] \\
& +\operatorname{tr}\left[f_{c} G_{y z}^{+} f_{d} G_{z x}^{+} f_{a} O_{x} f_{b} O_{y}^{T}\right] ;
\end{aligned}
$$

see [7] and Appendix A for details. Therefore we obtain for Eq. (21) the expression

$$
\begin{aligned}
v_{+}^{a} J_{a}^{+}= & -v_{+}^{a c l} O^{a b}\left(v_{+}^{c l}\right)\left(\partial_{i} \partial_{-} \rho_{b}^{i}\right) \\
& -\frac{1}{2} g \varepsilon_{+x}^{a}\left(U_{1}^{a b}\right)_{x y}^{c}\left(\partial_{i} \partial_{-} \rho_{b}^{i}\right)_{x} \varepsilon_{+y}^{c} .
\end{aligned}
$$

\section{One-loop effective action: integration over fluctuations}

We perform the computation of the one-loop correction to the effective action in light-cone gauge using a noncanonical method, integrating out subsequently transverse and longitudinal fluctuations. The reason for the use of this non-canonical method of the calculation is simple. The Lagrangian Eq. (1) consists of a new term in comparison to the usual gluon QCD Lagrangian. Consequently, instead of the canonical equation of motion which relates transverse and longitudinal fields, we have the following equation:

$-\left(D_{i}\left(\partial_{-} v^{i}\right)\right)_{a}-\partial_{-}^{2} v_{a+}=j_{a}^{+}\left(v_{+}\right) ;$

see [7]. This equation is different from the "canonical" one and will lead to a different constraint in the canonical quantization method; see [54,55]. Still, it is possible to make the usual substitution in the Lagrangian which relates these fields, see for example [55], and we define the canonical lightcone Lagrangian in the usual form in the limit $g \rightarrow 0$. But in that case we will make a shift in the argument of the effective current term, especially in light of the condition of Eq. (10), and in turn that will lead to some complicated expression of the induced current in the equations of motion to $g^{2}$ and higher orders of perturbative theory. Therefore, we prefer to use the non-canonical method of introducing bare propagators in the theory, calculating the final one-loop expressions in terms of these propagators; see Appendix B. As we shall see there, after the resummation of one-loop terms, the wellknown light-cone propagators, see for example [44-49,56], are arising in the expressions. So far it is not clear, whether it os a result of the chosen precision of the calculations or if it is a feature of the effective Lagrangian Eq. (1); we will investigate this question in a separate publication.

\subsection{Integration on transverse fluctuations}

Collecting quadratic terms for the transverse fluctuation and the effective current term we obtain

$$
\begin{aligned}
-\frac{1}{2} \varepsilon_{i}^{a}\left(\delta_{a c}\left(\delta_{i j} \square+\partial_{i} \partial_{j}\right)\right. & \\
& -2 g f_{a b c}\left(\delta_{i j}\left(v_{k}^{b c l} \partial_{k}-v_{+}^{b c l} \partial_{-}\right)\right. \\
& \left.-\frac{1}{2}\left(v_{j}^{b c l} \partial_{i}+v_{i}^{b c l} \partial_{j}-F_{i j}^{b}\right)\right) \\
& \left.-g^{2} f_{a b c_{1}} f_{c_{1} b_{1} c}\left(\delta_{i j} v_{k}^{b c l} v_{k}^{b_{1} c l}-v_{i}^{b c l} v_{j}^{b_{1} c l}\right)\right) \varepsilon_{j}^{c} \\
& +J_{i}^{a} \varepsilon_{i}^{a} \\
= & -\frac{1}{2} \varepsilon_{i}^{a}\left(\left(M_{0}\right)_{i j}^{a c}+\left(M_{1}\right)_{i j}^{a c}+\left(M_{2}\right)_{i j}^{a c}\right) \varepsilon_{j}^{c}+J_{i}^{a} \varepsilon_{i}^{a} .
\end{aligned}
$$

There are the following operators with respect to the transverse fluctuations, which we determine and which we will use in the further calculations. The first one reads

$\tilde{G}_{i j}^{a c}=\left[\left(M_{0}\right)_{i j}^{a c}+\left(M_{1}\right)_{i j}^{a c}+\left(M_{2}\right)_{i j}^{a c}\right]^{-1}$,

the second one is

$G_{i j}^{a c}=\left[\left(M_{0}\right)_{i j}^{a c}+\left(M_{1}\right)_{i j}^{a c}\right]^{-1}$,

and the third one, which is the bare propagator of the transverse fluctuations, is

$G_{0 i j}^{a c}=\left[\left(M_{0}\right)_{i j}^{a c}\right]^{-1}$.

We write the inverse operator expressions of Eqs. (32)-(32) in the following perturbative forms:

$$
\begin{aligned}
\tilde{G}_{i j}^{a c}(x, y)= & G_{0 i j}^{a c}(x, y)-\int \mathrm{d}^{4} z G_{0 i j^{\prime}}^{a b}(x, z)\left(\left(M_{1}(z)\right)_{j^{\prime} j^{\prime \prime}}^{b d}\right. \\
& \left.+\left(M_{2}(z)\right)_{j^{\prime} j^{\prime \prime}}^{b d}\right) \tilde{G}_{j^{\prime \prime} j}^{d c}(z, y)
\end{aligned}
$$

and

$$
\begin{aligned}
G_{i j}^{a c}(x, y)= & G_{0 i j}^{a c}(x, y) \\
& -\int \mathrm{d}^{4} z G_{0 i j^{\prime}}^{a b}(x, z)\left(M_{1}(z)\right)_{j^{\prime} j^{\prime \prime}}^{b d} G_{j^{\prime \prime} j}^{d c}(z, y),
\end{aligned}
$$


with the bare propagator defined through

$\left(M_{0}(x)\right)_{i j}^{a c} G_{0 j l}^{c b}(x, y)=\delta^{a b} \delta_{i l} \delta^{4}(x-y)$,

where

$\left(M_{0}\right)_{i j}^{a c}=\delta_{a c}\left(\delta_{i j} \square+\partial_{i} \partial_{j}\right)$.

The solution of Eq. (37) is simple:

$$
\begin{aligned}
G_{0 i j}^{a b}(x, y) & =-\delta^{a b} \int \frac{\mathrm{d}^{4} p}{(2 \pi)^{4}} \frac{e^{-l p(x-y)}}{p^{2}}\left(\delta_{i j}-\frac{p_{i} p_{j}}{2\left(p_{-} p_{+}\right)}\right) \\
& =-\delta^{a b} G_{0 i j}(x, y)
\end{aligned}
$$

and determines the above operators as a perturbative series based on Eqs. (35)-(36).

Integrating out the transverse fluctuation we obtain the following expression for the effective action:

$$
\begin{aligned}
\Gamma= & \int \mathrm{d}^{4} x\left(L_{Y M}\left(v_{i}^{c l}, v_{+}^{c l}, \varepsilon_{+}\right)-v_{+c l}^{a} J_{a}^{+}\left(v_{+}^{c l}\right)-A_{+}^{a}\left(\partial_{i}^{2} A_{-}^{a}\right)\right) \\
& +\frac{l}{2} \ln \left(1+G_{0} M_{1}\right)+\frac{l}{2} \ln \left(1+G M_{2}\right)+\frac{1}{2} \int \mathrm{d}^{4} x \int \mathrm{d}^{4} y \\
& \times\left(g \varepsilon_{+x}^{a}\left(U_{1}^{a b}\right)_{x y}^{c}\left(\partial_{i} \partial_{-} \rho_{b}^{i}\right)_{x} \varepsilon_{+y}^{c}+J_{i x}^{a} \tilde{G}_{i j}^{a b}(x, y) J_{j y}^{b}\right),
\end{aligned}
$$

with all reggeon field terms in the Lagrangian included.

\subsection{Integration on longitudinal fluctuations}

Collecting only quadratic terms for the longitudinal fluctuations we write the corresponding part of the action as

$$
\begin{aligned}
\Gamma_{\varepsilon_{+}^{2}}= & -\frac{1}{2} \int \mathrm{d}^{4} x \int \mathrm{d}^{4} y \varepsilon_{+x}^{a}\left(\left(N_{0}\right)_{a b}^{++}\right. \\
& \left.+\left(N_{1}\right)_{a b}^{++}+\left(N_{2}\right)_{a b}^{++}+\left(N_{3}\right)_{a b}^{++}\right)_{x y} \varepsilon_{+y}^{b},
\end{aligned}
$$

with the inverse propagator term

$\left(N_{0}\right)_{a b}^{++}=\delta_{x y} \delta^{a b} \partial_{-y}^{2}-\left(\partial_{-x} \partial_{-y} \partial_{i x} \partial_{j y} G_{0 i j}^{a b}(x, y)\right)$.

Correspondingly, the other terms in the Lagrangian of Eq. (41) are determined by the following expressions:

$$
\left(N_{1}\right)_{a b}^{++}=-g\left(U_{1}^{a c}\right)_{x y}^{b}\left(\partial_{i} \partial_{-} \rho_{c}^{i}\right)_{x},
$$

the third term,

$$
\begin{aligned}
\left(N_{2}\right)_{a b}^{++}= & -2 g f_{c d b}\left(\left(\partial_{-y} v_{j y}^{d c l}\right)\left(\partial_{-x} \partial_{i x} \tilde{G}_{i j}^{a c}(x, y)\right)\right. \\
& \left.-v_{j y}^{d c l}\left(\partial_{-y} \partial_{-x} \partial_{i x} \tilde{G}_{i j}^{a c}(x, y)\right)\right) \\
& -g^{2} f_{c c_{1} a} f_{d d_{1} b}\left(\left(\partial_{-} v_{i}^{c_{1} c l}\right)-v_{i}^{c_{1} c l} \partial_{-}\right)_{x} \\
& \times\left(\left(\partial_{-} v_{j}^{d_{1} c l}\right)-v_{j}^{d_{1} c l} \partial_{-}\right)_{y} \tilde{G}_{i j}^{c d}(x, y),
\end{aligned}
$$

and the last one,

$$
\left(N_{3}\right)_{a b}^{++}=-\left(\partial_{-} \partial_{i}\right)_{x}\left(\partial_{-} \partial_{j}\right)_{y}\left(\tilde{G}_{i j}^{a b}(x, y)-G_{0 i j}^{a b}(x, y)\right) .
$$

The term of Eq. (42) determines the equation for the longitudinal bare propagator:

$\int \mathrm{d}^{4} y N_{0 a b}^{++}(x, y) G_{0++}^{b c}(y, z)=\delta^{a c} \delta_{x z}$

with solution

$$
\begin{aligned}
G_{0++}^{a b}(x, y) & =-2 \delta^{a b} \int \frac{\mathrm{d}^{4} p}{(2 \pi)^{4}} \frac{e^{-\imath p(x-y)}}{p^{2}} \frac{p_{+}}{p_{-}} \\
& =-\delta^{a b} G_{0++}(x, y) .
\end{aligned}
$$

Therefore, integrating out this fluctuation, we obtain

$$
\begin{aligned}
\Gamma= & \int \mathrm{d}^{4} x\left(L_{Y M}\left(v_{i}^{c l}, v_{+}^{c l}\right)-v_{+c l}^{a} J_{a}^{+}\left(v_{+}^{c l}\right)-A_{+}^{a}\left(\partial_{i}^{2} A_{-}^{a}\right)\right) \\
& +\frac{l}{2} \ln \left(1+G_{0} M_{1}\right)+\frac{l}{2} \ln \left(1+G M_{2}\right) \\
& +\frac{l}{2} \ln \left(1+G_{0++}^{b a}\left(\left(N_{1}\right)_{a b}^{++}+\left(N_{2}\right)_{a b}^{++}+\left(N_{3}\right)_{a b}^{++}\right)\right),
\end{aligned}
$$

which is a functional of the reggeized gluon fields only.

\section{RFT calculus based on the effective action}

The construction of the RFT calculus based on the effective action of Eq. (48) requires knowledge of the classical solutions of Eq. (11) in terms of reggeon fields. This task was performed in [7], the classical solutions found are the following:

$$
\begin{aligned}
v_{+}^{a c l}= & A_{+}^{a}-2 g \square^{-1}\left[f_{a b c}\left(U^{b b_{1}}\left(A_{+}\right) \rho_{b_{1}}^{i}\right)\left(\partial_{i} A_{+}^{c}\right)\right] \\
& +4 g^{2} \square^{-1}\left[f_{a b c}\left(U^{b b_{1}}\left(A_{+}\right) \rho_{b_{1}}^{i}\right)\right. \\
& \left.\times \partial_{i}\left\{\square^{-1}\left[f_{c b_{2} c_{1}}\left(U^{b_{2} b_{3}}\left(A_{+}\right) \rho_{b_{3}}^{j}\right)\left(\partial_{j} A_{+}^{c_{1}}\right)\right]\right\}\right] \\
& =A_{+}^{a}+g \Phi_{+1}^{a}\left(A_{+}\right)+g^{2} \Phi_{+2}^{a}\left(A_{+}\right)
\end{aligned}
$$

and

$$
\begin{aligned}
v_{i}^{a c l}= & v_{i 0}^{a}+g v_{i 1}^{a}=U^{a b}\left(v_{+}^{c l}\right) \rho_{b i}\left(x^{-}, x_{\perp}\right) \\
& -g\left[\square^{-1}\left(\partial^{j} P_{j i}^{a}+\frac{1}{g} \partial_{i}\left(\left(\partial^{j} U^{a b}\right) \rho_{j}^{b}\right)+\partial_{i} \partial_{-}^{-1} j_{a 1}^{+}\right)\right] \\
= & U^{a b}\left(v_{+}^{c l}\right) \rho_{b i}+g \Lambda_{i 1}^{a}\left(A_{+}\right),
\end{aligned}
$$

with some complicated $P_{j i}$ function; see [7], and

$j_{a 1}^{+}=f_{a b c} v_{j 0}^{b}\left(\partial_{-} v_{0}^{j c}\right)$.

On the base of these solutions, the effective action of Eq. (48) can be written as a functional of the reggeized fields only. We have

$$
\begin{aligned}
\left(F_{i+}^{a} F_{i-}^{a}\right)_{c l} & =\left(\partial_{-} v_{i}^{a}\right)\left(D_{+} v_{i}\right)^{a}-\left(\partial_{-} v_{i}^{a}\right)\left(\partial_{i} v_{+}^{a}\right) \\
& =v_{+}^{a}\left(D_{i}\left(\partial_{-} v_{i}\right)\right)^{a}+\left(\partial_{-} v_{i}^{a}\right)\left(\partial_{+} v_{i}^{a}\right)
\end{aligned}
$$


Taking into account an identity from the equation of motion

$\left(D_{i}\left(\partial_{-} v_{i}\right)\right)^{a}=\partial_{-}^{2} v_{+}^{a}+U^{a b}\left(v_{+}\right)\left(\partial_{-} \partial_{i} \rho_{i}^{b}\right)$,

we obtain to $g^{2}$ accuracy

$$
\begin{aligned}
\left(F_{i+}^{a} F_{i-}^{a}\right)_{c l}= & -g^{2}\left(\partial_{-} \Phi_{+1}^{a}\right)\left(\partial_{-} \Phi_{+1}^{a}\right) \\
& +v_{+}^{a c l} U^{a b}\left(A_{+}\right)\left(\partial_{-} \partial_{i} \rho_{i}^{b}\right)+\left(\partial_{-} v_{i}^{a c l}\right)\left(\partial_{+} v_{i}^{a c l}\right) .
\end{aligned}
$$

Correspondingly, there are also the following terms of the "net" effective action:

$$
\begin{aligned}
\frac{1}{2}\left(F_{+-}^{a} F_{+-}^{a}\right)_{c l}= & \frac{1}{2} g^{2}\left(\partial_{-} \Phi_{+1}^{a}\right)\left(\partial_{-} \Phi_{+1}^{a}\right) \\
= & 2 g^{2} \square^{-1}\left[f_{a b c}\left(U^{b d}\left(A_{+}\right)\left(\partial_{-} \rho_{d}^{i}\right)\right)\left(\partial_{i} A_{+}^{c}\right)\right] \square^{-1} \\
& \times\left[f_{a b_{1} c_{1}}\left(U^{b_{1} d_{1}}\left(A_{+}\right)\left(\partial_{-} \rho_{d_{1}}^{j}\right)\right)\left(\partial_{j} A_{+}^{c_{1}}\right)\right]
\end{aligned}
$$

and

$-\frac{1}{4}\left(F_{i j}^{a} F_{i j}^{a}\right)_{c l}=-\frac{1}{4}\left(F_{i j}^{a}\right)_{c l 0}\left(F_{i j}^{a}\right)_{c l 0}$

with

$$
\begin{aligned}
\left(F_{i j}^{a}\right)_{c l 0}= & \rho_{b j} \partial_{i} U^{a b}\left(v_{+0}^{c l}\right)-\rho_{b i} \partial_{j} U^{a b}\left(v_{+0}^{c l}\right) \\
& +g f_{a b c}\left(U^{b b_{1}}\left(v_{+0}^{c l}\right) \rho_{b_{1} i}\right)\left(U^{c c_{1}}\left(v_{+0}^{c l}\right) \rho_{c_{1} j}\right) \\
& +g\left(\partial_{i} \Lambda_{j 1}^{a}-\partial_{j} \Lambda_{i 1}^{a}\right) \\
& +g^{2} f_{a b c}\left(U^{b b_{1}} \rho_{b_{1} i} \Lambda_{j 1}^{c}+\Lambda_{i 1}^{b} U^{c c_{1}} \rho_{c_{1} j}\right)
\end{aligned}
$$

where we notice that Eq. (56)'s minimal order is $g^{2}$. Now, based on the connection between the $A_{-}$field and the $\rho_{i}$ operator

$\partial_{i} \partial_{-} \rho_{a}^{i}=-\frac{1}{N} \partial_{i}^{2} A_{-}$,

or

$\rho_{i}^{a}\left(x^{-}, x_{\perp}\right)=\frac{1}{N} \int \mathrm{d}^{4} z G_{x z}^{-0}\left(\partial_{i z} A_{-}^{a}\left(z^{-}, z_{\perp}\right)\right)$,

see [7] and Appendix A, the effective action of Eq. (48) can be expanded in terms of reggeon fields $A_{-}$and $A_{+}$as

$\Gamma=\sum_{n, m=0}\left(A_{+}^{a_{1}} \cdots A_{+}^{a_{n}} K_{b_{1} \cdots b_{m}}^{a_{1} \cdots a_{n}} A_{-}^{b_{1}} \cdots A_{-}^{b_{m}}\right)$,

that determines this expression as a functional of the reggeon fields and provides effective vertices of the interactions of the reggeized gluons in the RFT calculus.

\section{Interaction kernels and propagators of reggeized gluons}

The effective action of Eq. (60) can be fully determined in terms of the effective vertices of reggeon fields interactions.
Calculating these vertices one after another we will reconstruct the expression similar to introduced in [8], see also [57-68], which can be considered as QCD Hamiltonian for reggeized gluon fields. There are the following well-known vertices of the interactions of reggeon fields. These are vertices of the interaction of $A_{+}$and $A_{-}$fields and the vertex of the interaction of two $A_{+}$fields and two $A_{-}$, which are propagators of reggeized gluons and BFKL kernel, respectively, see [33-38], the vertex of the interaction of six reggeon fields, which can be identified with a triple Pomeron vertex, see [39-41], or with an odderon one; see [69-77]. Equation (48) consists of these vertices plus many others with different precision and different color representations. Calculating the QFT corrections to this effective action we as well will calculate the corrections to these vertices and will determine the expressions for other complex vertices of interactions of reggeized fields in RFT. Anyway, a recalculation of the known vertices is a good test of the self-consistency of the effective action. Therefore, in this paper we calculate the propagator of reggeized gluons, which is the basic element of the small-x BFKL approach, in the framework of the effective action for reggeized gluons.

The interaction of reggeized gluons $A_{+}$and $A_{-}$is defined as an effective vertex of interactions of reggeon fields in Eq. (60):

$\left(K_{x y}^{a b}\right)^{+-}=K_{x y}^{a b}=\left(\frac{\delta^{2} \Gamma}{\delta A_{+x}^{a} \delta A_{-y}^{b}}\right)_{A_{+}, A_{-}=0}$,

we can call this vertex as interaction kernel as well; see Eqs. (68)-(71) below. The contributions to this kernel are provided by the different terms in the action which are linear with respect to the $A_{+}, A_{-}$fields. Namely, the variation of the logarithms in Eq. (48) gives

$$
\begin{aligned}
-2 \imath & K_{x y}^{a b}=\left(\frac{\delta^{2} \ln (1+G M)}{\delta A_{+x}^{a} \delta A_{-y}^{b}}\right)_{A_{+}, A_{-}=0} \\
= & {\left[\left(\frac{\delta^{2} G}{\delta A_{+x}^{a} \delta A_{-y}^{b}} M+\frac{\delta G}{\delta A_{+x}^{a}} \frac{\delta M}{\delta A_{-y}^{b}}+\frac{\delta G}{\delta A_{-y}^{b}} \frac{\delta M}{\delta A_{+x}^{a}}\right.\right.} \\
& \left.+G \frac{\delta^{2} M}{\delta A_{+x}^{a} \delta A_{-y}^{b}}\right) \times(1+G M)^{-1} \\
& -\left(\frac{\delta G}{\delta A_{-y}^{b}} M+G \frac{\delta M}{\delta A_{-y}^{b}}\right)(1+G M)^{-1} \\
& \left.\times\left(\frac{\delta G}{\delta A_{+x}^{a}} M+G \frac{\delta M}{\delta A_{+x}^{a}}\right)(1+G M)^{-1}\right]_{A_{+}, A_{-}=0}
\end{aligned}
$$

see the expression of the effective action of Eq. (48).

Therefore, we have the following contributions in the kernel. The leading order contribution to $K_{x y 0}^{a b}$ is given by the 
second term in Eq. (54) and reads ${ }^{3}$

$K_{x y 0}^{a b}=-\delta^{a b} \delta_{x y} \partial_{i x}^{2}=\delta^{a b} \delta_{x y}\left(\partial_{i} \partial^{i}\right)_{x}$.

The NLO and NNLO contributions are determined by the logarithms in the r.h.s. of Eq. (48). Further the variation of the $\rho$ field with respect to $A_{-}$field will be used as well, and we have from Eq. (59)

$\frac{\delta \rho_{i}^{a}\left(x^{-}, x_{\perp}\right)}{\delta A_{-}^{b}\left(y^{-}, y_{\perp}\right)}=\frac{\delta^{a b}}{N} \int \mathrm{d}^{4} z G_{x z}^{-0} \delta\left(y^{-}-z^{-}\right) \delta^{2}\left(y_{\perp}-z_{\perp}\right) \partial_{i z}$.

Taking into account that

$G_{x z}^{-0}=\tilde{G}_{x^{-} z^{-}}^{-0} \delta\left(x^{+}-z^{+}\right) \delta^{2}\left(x_{\perp}-z_{\perp}\right)$,

we write Eq. (64) in the following form:

$\frac{\delta \rho_{i}^{a}\left(x^{-}, x_{\perp}\right)}{\delta A_{-}^{b}\left(y^{-}, y_{\perp}\right)}=\frac{1}{N} \delta^{a b} \delta^{2}\left(x_{\perp}-y_{\perp}\right) \tilde{G}_{x^{-} y^{-}}^{-0} \partial_{i y}$,

where the regularization of the pole of the $\tilde{G}_{x^{-} y^{-}}^{-0}$ Green's function depends on its definition; see Appendix A.

The propagator corresponding to the kernel of Eq. (61) is defined as a solution of the following equation:

$\int \mathrm{d}^{4} z\left(K_{x z}^{a b}\right)^{-+}\left(D_{z y}^{b c}\right)_{+-}=\delta^{a c} \delta_{x y}$.

For the propagator of the reggeized gluons, $D_{+-}$, the following perturbative series can be defined therefore:

$$
\begin{aligned}
\left(D_{x y}^{a c}\right)_{+-}= & \left(D_{x y}^{a c}\right)_{0+-} \\
& -\int \mathrm{d}^{4} z \int \mathrm{d}^{4} w\left(D_{x z}^{a b}\right)_{0+-}\left(\left(K_{z w}^{b d}\right)^{-+}\right. \\
& \left.-\left(K_{z w}^{b d}\right)_{0}^{-+}\right)\left(D_{w y}^{w c}\right)_{+-},
\end{aligned}
$$

or in brief notation

$D_{x y}^{a c}=D_{x y 0}^{a c}-\int \mathrm{d}^{4} z \int \mathrm{d}^{4} w D_{x z 0}^{a b}\left(K_{z w}^{b d}-K_{z w 0}^{b d}\right) D_{w y}^{w c}$,

where

$K_{z w}^{b d}=\sum_{k=0} K_{z w k}^{b d}$

and

$\int \mathrm{d}^{4} z K_{x z 0}^{a b} D_{z y 0}^{b c}=\delta^{a c} \delta_{x y}$.

$\overline{3}$ We note also that there are also different kernel, related to $\left\langle A_{+} A_{+}\right\rangle$ and $\left\langle A_{-} A_{-}\right\rangle$propagators, in the approach. In leading order the contributions to these kernels are zero:

$\left(K_{x y}^{a b}\right)_{0}^{++}=\left(K_{x y}^{a b}\right)_{0}^{--}=0$

we do not calculate these propagators in the paper. This task will be considered in a separate publication.
The calculation of the NLO kernel $K_{x y 1}^{a b}$ is performed in Appendix B; the following answer is obtained:

$$
\begin{aligned}
& -2 \imath K_{x y 1}^{a b}=\frac{1}{2} g^{2} N \delta^{a b} \int \mathrm{d}^{4} z \mathrm{~d}^{4} t \mathrm{~d}^{4} w\left(\partial_{i z}^{2} G_{0++}^{t z}\right) \\
& \times\left(\delta_{x_{\perp} w_{\perp}}^{2} \delta_{x^{+} w^{+}}\right)\left(\delta_{y_{\perp} z_{\perp}}^{2} \delta_{y^{-} z^{-}}\right) \cdot\left[\left(G_{z w}^{+0} G_{w t}^{+0}+G_{t w}^{+0} G_{w z}^{+0}\right)\right. \\
& \left.+2\left(G_{z t}^{+0} G_{t w}^{+0}+G_{z t}^{+0} G_{w z}^{+0}+G_{z w}^{+0} G_{t z}^{+0}+G_{t z}^{+0} G_{w t}^{+0}\right)\right] \\
& -\int \mathrm{d}^{4} z \mathrm{~d}^{4} t \hat{G}_{i j}^{z t}\left(\frac{\delta}{\delta A_{+x}^{a}}\left(M_{1 j j_{1}}^{c d}\right)_{t}\right) \hat{G}_{j_{i} j_{2}}^{t z}\left(\frac{\delta}{\delta A_{-y}^{b}}\left(M_{1 j_{2} i}^{d c}\right)_{z}\right) \\
& -\frac{1}{2} g f_{c d b} \int \mathrm{d}^{4} w \mathrm{~d}^{4} t \mathrm{~d}^{4} w_{1}\left(G_{t w}^{+0}-G_{w t}^{+0}\right)\left(\delta_{t_{\perp} y_{\perp}}^{2} \delta_{t^{-} y^{-}}\right) \\
& \times \hat{G}_{+j_{1}}^{w w_{1}}\left(\frac{\delta}{\delta A_{+x}^{a}}\left(M_{1 j_{1} j_{2}}^{d c}\right)_{w_{1}}\right)\left(\partial_{i t}^{2} \hat{G}_{j_{2}+}^{w_{1} t}\right) \\
& +2 g f_{c d_{1} d} \int \mathrm{d}^{4} w \mathrm{~d}^{4} w_{1} \hat{G}_{+j_{1}}^{w w_{1}}\left(\frac{\delta}{\delta A_{+x}^{a}}\left(M_{1 j_{1} j_{2}}^{d c}\right)_{w_{1}}\right) \\
& \times\left(\frac{\delta}{\delta A_{-y}^{b}}\left(\left(\partial_{-} v_{j}^{d_{1} c l}\right)-v_{j}^{d_{1} c l} \partial_{-}\right)_{w}\right) \hat{G}_{j_{2} j}^{w_{1} w},
\end{aligned}
$$

where the expressions for all functional derivatives are presented in Appendix B. Nevertheless, the only non-zero contributions to this kernel are the following:

$$
\begin{aligned}
& -2 \imath K_{x y 1}^{a b}=\frac{1}{2} g^{2} N \delta^{a b} \int \mathrm{d}^{4} z \mathrm{~d}^{4} t \mathrm{~d}^{4} w\left(\partial_{i z}^{2} G_{0++}^{t z}\right) \\
& \times\left(\delta_{x_{\perp} w_{\perp}}^{2} \delta_{x^{+} w^{+}}\right)\left(\delta_{y_{\perp} z_{\perp}}^{2} \delta_{y^{-} z^{-}}\right) G_{t w}^{+0} G_{w z}^{+0} \\
& +\frac{1}{2} g f_{c d b} \int \mathrm{d}^{4} w \mathrm{~d}^{4} t \mathrm{~d}^{4} w_{1} G_{w t}^{+0}\left(\delta_{t_{\perp} y_{\perp}}^{2} \delta_{t^{-} y^{-}}\right) \\
& \times \hat{G}_{+j_{1}}^{w w_{1}}\left(\frac{\delta}{\delta A_{+x}^{a}}\left(M_{1 j_{1} j_{2}}^{d c}\right)_{w_{1}}\right)\left(\partial_{i t}^{2} \hat{G}_{j_{2}+}^{w_{1} t}\right) ;
\end{aligned}
$$

see Appendix C. Therefore, using Eq. (C.7) and Eq. (C.22) we have

$$
\begin{aligned}
-2 \imath K_{x y 1}^{a b}= & \frac{\imath g^{2} N}{4 \pi} \partial_{i x}^{2}\left(\int \frac{\mathrm{d} p_{-}}{p_{-}} \int \frac{\mathrm{d}^{2} p_{\perp}}{(2 \pi)^{2}}\right. \\
& \left.\times \int \frac{\mathrm{d}^{2} k_{\perp}}{(2 \pi)^{2}} \frac{k_{\perp}^{2}}{p_{\perp}^{2}\left(p_{\perp}-k_{\perp}\right)^{2}} e^{-\imath k_{i}\left(x_{i}-y_{i}\right)}\right)
\end{aligned}
$$

and performing the Fourier transform we write Eq. (69) as

$\tilde{D}^{a b}(p)=\frac{\delta^{a b}}{p_{\perp}^{2}}-\frac{g^{2} N}{32 \pi^{3}} \int \frac{\mathrm{d} k_{-}}{k_{-}} \int \mathrm{d}^{2} k_{\perp} \frac{p_{\perp}^{2}}{k_{\perp}^{2}\left(p_{\perp}-k_{\perp}\right)^{2}} \tilde{D}^{a b}(p)$,

where we used

$D_{0}^{a b}(x, y)=\delta^{a b} \int \frac{\mathrm{d}^{4} p}{(2 \pi)^{4}} \frac{e^{-l p(x-y)}}{p_{\perp}^{2}}$,

see the definitions of Eqs. (63) and (71). Introducing the rapidity variable $y=\frac{1}{2} \ln \left(\Lambda k_{-}\right)$and taking into account the physical cut-off of the rapidity related with particles cluster size $\eta$, we obtain after integration on the variable $k_{-}$in the 
limits $y_{0}-\eta / 2$ and $y_{0}+\eta / 2$

$$
\begin{aligned}
\tilde{D}^{a b}(p \perp, \eta)= & \frac{\delta^{a b}}{p_{\perp}^{2}}-\frac{g^{2} N}{16 \pi^{3}} \int_{0}^{\eta} d \eta^{\prime} \int \mathrm{d}^{2} k_{\perp} \frac{p_{\perp}^{2}}{k_{\perp}^{2}\left(p_{\perp}-k_{\perp}\right)^{2}} \\
& \tilde{D}^{a b}\left(p_{\perp}, \eta^{\prime}\right)
\end{aligned}
$$

with

$\epsilon\left(p_{\perp}^{2}\right)=-\frac{\alpha_{s} N}{4 \pi^{2}} \int \mathrm{d}^{2} k_{\perp} \frac{p_{\perp}^{2}}{k_{\perp}^{2}\left(p_{\perp}-k_{\perp}\right)^{2}}$

as intercept of the propagator of reggeized gluons. Rewriting this equation as a differential one:

$\frac{\partial \tilde{D}^{a b}(p)}{\partial \eta}=\tilde{D}^{a b}(p) \epsilon\left(p_{\perp}^{2}\right)$

we obtain the final expression for the propagator:

$\tilde{D}^{a b}(p)=\frac{\delta^{a b}}{p_{\perp}^{2}}\left(\frac{s}{s_{0}}\right)^{\epsilon\left(p_{\perp}^{2}\right)}$

with some rapidity interval $0<\eta<\ln \left(s / s_{0}\right)$ introduced of the problem of interest. We note that the propagator obtained is precisely the well-known one, see [33-38], and thereby we demonstrated the self-consistency of the obtained effective action of Eq. (48). It is interesting to note also that the obtained intercept, Eq. (78), is known as well in CGC approach, this is a color charge density; see for example the work of Kovner et al. in [44-49].

\section{Conclusion}

The main result of this paper is the expression for the oneloop effective action for reggeized gluons in Eq. (48). On one hand, this effective action can be considered as the one-loop effective action for gluodynamics with added gauge invariant source of longitudinal gluons, calculated on the base of non-trivial classical solutions for gluon fields. These classical solutions are fully determined in terms of reggeized gluons fields, and, therefore, the same action can be considered as the one-loop effective action for reggeized gluons. The expansion of this action in terms of the reggeons, see Eq. (60), determines the vertices of the interactions of these reggeized fields. There are all possible vertices in here, the only limitation is the precision of Eq. (48). Whereas the $A_{+}$ reggeized field is the argument of the ordered exponential in the classical solutions and the number of derivatives with respect to this field is not limited, see Eqs. (49)-(50) and [7], the $A_{-}$reggeon field is presented in Eq. (48) in a combination which allows only a limited number of derivations with respect to this field. Therefore, the calculation of the complex vertices related to large number of $A_{-}$reggeized fields will require or increase of the precision of the calculations, or calculation of the same effective action in a different gauge, where two types of the ordered exponential, with $A_{+}$ and $A_{-}$fields in arguments, will be presented in the classical solutions. In both cases, the precision of the computations will be determined by the QFT methods, namely it will be limited by the orders of the classical solutions, order of loops included in calculations and by combinations of the fields in the final expression, which will survive after application of the $A_{+}, A_{-} \rightarrow 0$ limit.

Another important result of the paper is the calculation of the propagator of reggeized gluons of Eq. (80) in the framework of the approach. Although this propagator is well known and widely used in all applications of the effective action, see [9-22] and [33-38], the full computation of the propagator in the framework of interest was done for the first time. We can consider this calculation as a check of the selfconsistency of the approach and also as an explanation of the methods of the calculation of small-x BFKL-based vertices in the framework. There are other important vertices which can be similarly calculated based on Eq. (48). These vertices are important ingredients of the unitary corrections to different production and interaction amplitudes of the processes at high energies and they will be considered in separate publications.

As we mentioned above, Eq. (48) describes the interactions of the reggeized gluons inside a cluster of particles in a limited range of rapidity. Therefore, following [8], we can define this expression as an RFT Hamiltonian written in terms of the QCD reggeized gluons. The performed calculations, in turn, can be considered as a construction of QCD RFT in terms of QCD degrees of freedom. This RFT construction is interesting because it allows one to consider the field theory in terms of $A_{-}$and $A_{+}$fields only, developing an approach to the calculation of reggeon loops, applications of the Hamiltonian in the integrable systems frameworks and in condensed matter physics, [78-80], and use of the methods in the effective gravity approach, see [1-6].

In conclusion we emphasize that the paper is considered as an additional step to developing of the effective theory for reggeized gluons, which will be useful in a variety of applications in high energy physics and other research fields.

Open Access This article is distributed under the terms of the Creative Commons Attribution 4.0 International License (http://creativecomm ons.org/licenses/by/4.0/), which permits unrestricted use, distribution, and reproduction in any medium, provided you give appropriate credit to the original author(s) and the source, provide a link to the Creative Commons license, and indicate if changes were made.

Funded by $\mathrm{SCOAP}^{3}$. 


\section{Appendix A: Representation and properties of $O$ and $O^{T}$ operators}

For the arbitrary representation of the gauge field $v_{+}=$ $\imath T^{a} v_{+}^{a}$ with $D_{+}=\partial_{+}-g v_{+}$, we can consider the following representation of $O$ and $O^{T}$ operators ${ }^{4}$ :

$$
\begin{aligned}
O_{x} & =\delta^{a b}+g \int \mathrm{d}^{4} y G_{x y}^{+a a_{1}}\left(v_{+}(y)\right)_{a_{1} b} \\
& =1+g G_{x y}^{+} v_{+y}
\end{aligned}
$$

and correspondingly

$O_{x}^{T}=1+g v_{+y} G_{y x}^{+}$,

which is a redefinition of the operator expansions used in [16] in terms of Green's function instead of integral operators; see [7] for more details. The Green's function in the above equations we understand as the Green's function of the $D_{+}$ operator and express it in the perturbative sense by

$G_{x y}^{+}=G_{x y}^{+0}+g G_{x z}^{+0} v_{+z} G_{z y}^{+}$

and

$G_{y x}^{+}=G_{y x}^{+0}+g G_{y z}^{+} v_{+z} G_{z x}^{+0}$,

with the bare propagators defined as (there is no integration on the variable $x$ )

$\partial_{+x} G_{x y}^{+0}=\delta_{x y}, G_{y x}^{+0} \overleftarrow{\partial}_{+x}=-\delta_{x y}$.

The following properties of the operators can be derived:

1.

$$
\begin{aligned}
\delta G_{x y}^{+} & =g G_{x z}^{+0}\left(\delta v_{+z}\right) G_{z y}^{+}+G_{x z}^{+0} v_{+z} \delta G_{z y}^{+} \\
& =g G_{x z}^{+0}\left(\delta v_{+z}\right) G_{z y}^{+}+G_{x z}^{+0} v_{+z}\left(\delta G_{z p}^{+}\right) D_{+p} G_{p y}^{+} \\
& =g\left(G_{x z}^{+0}\left(\delta v_{+z}\right) G_{z y}^{+}-G_{x z}^{+0} v_{+z} G_{z p}^{+}\left(\delta D_{+p}\right) G_{p y}^{+}\right) \\
& =g\left(G_{x p}^{+0}+G_{x z}^{+0} v_{+z} G_{z p}^{+}\right) \delta v_{+p} G_{p y}^{+} \\
& =g G_{x p}^{+} \delta v_{+p} G_{p y}^{+} ;
\end{aligned}
$$

2.

$$
\begin{aligned}
\delta O_{x} & =g G_{x y}^{+}\left(\delta v_{+y}\right)+g\left(\delta G_{x y}^{+}\right) v_{+y} \\
& =g G_{x p}^{+} \delta v_{+p}\left(1+g G_{p y}^{+} v_{+y}\right)=g G_{x p}^{+} \delta v_{+p} O_{p}
\end{aligned}
$$

\footnotetext{
${ }^{4}$ Due the light-cone gauge we consider here only $O\left(x^{+}\right)$operators. The construction of the representation of the $O\left(x^{-}\right)$operators can be done similarly.
}

3.

$$
\begin{aligned}
\partial_{+x} \delta O_{x} & =g\left(\partial_{+x} G_{x p}^{+}\right) \delta v_{+p} O_{p} \\
& =g\left(1+g v_{+x} G_{x p}^{+}\right) \delta v_{+p} O_{p}=g O_{x}^{T} \delta v_{+x} O_{x}
\end{aligned}
$$

4.

$$
\begin{aligned}
\partial_{+x} O_{x} & =g\left(\partial_{+x} G_{x y}^{+}\right) v_{+y}=g v_{+x}\left(1+g G_{x y}^{+} v_{+y}\right) \\
& =g v_{+x} O_{x}
\end{aligned}
$$

5.

$$
\begin{aligned}
O_{x}^{T} \overleftarrow{\partial}_{+x} & =g v_{+y}\left(G_{y x}^{+} \overleftarrow{\partial}_{+x}\right)=-g\left(1+v_{+y} G_{y x}^{+}\right) v_{+x} \\
& =-g O_{x}^{T} v_{+x}
\end{aligned}
$$

We see that the operator $O$ and $O^{T}$ have the properties of ordered exponents. For example, choosing bare propagators as

$G_{x y}^{+0}=\theta\left(x^{+}-y^{+}\right) \delta_{x y}^{3}, G_{y x}^{+0}=\theta\left(y^{+}-x^{+}\right) \delta_{x y}^{3}$,

we immediately reproduce

$O_{x}=P e^{g \int_{-\infty}^{x^{+}} d x^{\prime}+v_{+}\left(x^{\prime}+\right)}, O_{x}^{T}=P e^{g \int_{x^{+}}^{\infty} d x^{\prime}+v_{+}\left(x^{\prime}+\right)}$.

The form of the bare propagators which corresponds to another possible integral operator will lead to the more complicated representations of the $O$ and $O^{T}$ operators; see [16].

Now we consider a variation of the action's full current:

$$
\begin{aligned}
\delta \operatorname{tr}\left[v_{+x} O_{x} \partial_{i}^{2} A^{+}\right] & =\frac{1}{g} \delta \operatorname{tr}\left[\left(\partial_{+x} O_{x}\right) \partial_{i}^{2} A^{+}\right] \\
& =\frac{1}{g} \operatorname{tr}\left[\left(\partial_{+x} \delta O_{x}\right) \partial_{i}^{2} A^{+}\right] \\
& =\operatorname{tr}\left[O_{x}^{T} \delta v_{+x} O_{x}\left(\partial_{i}^{2} A^{+}\right)\right],
\end{aligned}
$$

which can be rewritten in the familiar form of Lipatov's induced current used in the paper:

$$
\begin{aligned}
\delta\left(v_{+} J^{+}\right) & =\delta \operatorname{tr}\left[\left(v_{+x} O_{x} \partial_{i}^{2} A^{+}\right)\right] \\
& =\delta v_{+}^{a} \operatorname{tr}\left[T_{a} O T_{b} O^{T}\right]\left(\partial_{i}^{2} A_{b}^{+}\right) .
\end{aligned}
$$

We note also that with the help of Eq. (A.1) for the representation of the $O$ operator the full action's current can we written as

$$
\begin{aligned}
& \operatorname{tr}\left[\left(v_{+x} O_{x}-A_{+}\right) \partial_{i}^{2} A^{+}\right] \\
& \quad=\operatorname{tr}\left[\left(v_{+}-A_{+}+v_{+x} G_{x y}^{+} v_{+y}\right)\left(\partial_{i}^{2} A^{+}\right)\right] .
\end{aligned}
$$




\section{Appendix B: Calculation of $K_{x y 1}^{a b}$ effective kernel}

Below we present all contributions to the vertex of interest and present the general answer for the kernel. We note that there are new effective propagators which arise in the calculations, see Eqs. (B.22) and (B.30)-(B.31) below. Introducing these propagators the effective resummation of the different contributions occurs, see Eqs. (B.23) and (B.37). This resummation can be performed directly in the expansion of logarithms in Eq. (48), before taking the derivatives. Whether these redefinitions of the propagators are valid for all order contributions, i.e. whether it can be done on the level of the Lagrangian is a subject of a separate publication. We do not consider this problem in the present paper.

\section{Transverse loop terms contributions}

First of all, consider the $M_{2}$ term in Eq. (48) to $g^{2}$ accuracy:

$$
\left(M_{2}\right)_{i j}^{a b}=-g^{2} f_{a c c_{1}} f_{c_{1} c_{2} b}\left(\delta_{i j} v_{k 0}^{c c l} v_{k 0}^{c_{2} c l}-v_{i 0}^{c c l} v_{j 0}^{c_{2} c l}\right) .
$$

We note that this term is quadratic with respect to the $\rho$ field and therefore it does not contribute to the kernel of interest.

For the $M_{1}$ term in Eq. (48) we have

$$
\begin{aligned}
& -2 l\left(K_{x y 1}^{a b}\right)_{1}=\left[G_{0} \frac{\delta^{2}\left(M_{1}^{c l}\right)^{c c}}{\delta A_{+x}^{a} \delta A_{-y}^{b}}\left(1+G_{0} M_{1}\right)^{-1}\right. \\
& \left.-G_{0} \frac{\delta\left(M_{1}^{c l}\right)^{c d}}{\delta A_{-y}^{b}}\left(1+G_{0} M_{1}\right)^{-1} G_{0} \frac{\delta\left(M_{1}^{c l}\right)^{d c}}{\delta A_{+x}^{a}}\left(1+G_{0} M_{1}\right)^{-1}\right]_{A_{+}, A_{-}=0} .
\end{aligned}
$$

Taking into account that

$$
\begin{aligned}
M_{1 i j}^{a b}= & -2 g f_{a c b}\left(\delta_{i j}\left(v_{k}^{c c l} \partial_{k}-v_{+}^{c c l} \partial_{-}\right)\right. \\
& \left.-\frac{1}{2}\left(v_{j}^{c c l} \partial_{i}+v_{i}^{c c l} \partial_{j}-\left(F_{i j}^{c}\right)_{c l}\right)\right),
\end{aligned}
$$

which with the required accuracy reads

$$
\begin{aligned}
M_{1 i j}^{a b}= & -2 g f_{a c b} \tilde{M}_{1 i j}^{c c l}=-2 g f_{a c b}\left(U^{c c_{1}} \delta_{i j} \rho_{k}^{c_{1}} \partial_{k}\right. \\
& \left.-\frac{1}{2} U^{c c_{1}}\left(\rho_{j}^{c_{1}} \partial_{i}+\rho_{i}^{c_{1}} \partial_{j}\right)-\delta_{i j} A_{+}^{c} \partial_{-}\right),
\end{aligned}
$$

we obtain

$$
\begin{aligned}
-2 l\left(K_{x y 1}^{a b}\right)_{1}= & -\int \mathrm{d}^{4} z \mathrm{~d}^{4} t\left(\frac{\delta}{\delta A_{-y}^{b}}\left(M_{1 i j}^{c d}\right)_{z}\right) G_{0 j k}(z, t) \\
& \times\left(\frac{\delta}{\delta A_{+x}^{a}}\left(M_{1 k l}^{d c}\right)_{t}\right) G_{0 l i}(t, z) .
\end{aligned}
$$

\section{Longitudinal loop terms contribution}

A few contributions arise from the last logarithm in Eq. (48). We account for only non-zero ones and calculate them one by one.

\section{$\left(N_{1}\right)_{a b}^{++}$term contribution}

The variation of the first term of the last logarithm in Eq. (48) gives

$$
\begin{aligned}
-2 l\left(K_{x y 1}^{a b}\right)_{2}= & {\left[G_{0++} \frac{\delta^{2}\left(N_{1}\right)_{c c}^{++}}{\delta A_{+x}^{a} \delta A_{-y}^{b}}\left(1+G_{0++} N_{1}^{++}\right)^{-1}\right.} \\
& -G_{0++} \frac{\delta\left(N_{1}\right)_{c d}^{++}}{\delta A_{-y}^{b}}\left(1+G_{0++} N_{1}^{++}\right)^{-1} G_{0++} \\
& \left.\times \frac{\delta\left(N_{1}\right)_{d c}^{++}}{\delta A_{+x}^{a}}\left(1+G_{0++} N_{1}^{++}\right)^{-1}\right]_{A_{+}, A_{-}=0},
\end{aligned}
$$

where we used $G_{0++}^{a b} \rightarrow \delta^{a b} G_{0++}$ definition. The $N_{1}$ term, which is determined by Eq. (43), is quadratic with respect to the reggeon fields, therefore only the first term in Eq. (B.6) gives the non-zero contribution:

$$
-2 \imath\left(K_{x y 1}^{a b}\right)_{2}=\left[G_{0++} \frac{\delta^{2}\left(N_{1}\right)_{c c}^{++}}{\delta A_{+x}^{a} \delta A_{-y}^{b}}\right]_{A_{+}, A_{-}=0} .
$$

We have

$$
\begin{aligned}
\frac{\delta^{2}\left(N_{1}\right)_{c c}^{++}}{\delta A_{+x}^{a} \delta A_{-y}^{b}} & =\frac{g}{N} \frac{\delta\left(U_{1}^{c d}\right)_{z t}^{c}}{\delta A_{+x}^{a}} \frac{\delta \partial_{i}^{2} A_{-z}^{d}}{\delta A_{-y}^{b}} \\
& =\frac{g^{2}}{N}\left(U_{2}^{c b}\right)_{z t w}^{c a_{1}} \frac{\delta v_{+w}^{a_{1} c l}}{\delta A_{+x}^{a}}\left(\delta_{y_{\perp} z_{\perp}}^{2} \delta_{y^{-} z^{-}}\right) \partial_{i z}^{2}
\end{aligned}
$$

At required accuracy we have

$$
\frac{\delta v_{+w}^{a_{1} c l}}{\delta A_{+x}^{a}}=\delta^{a a_{1}}\left(\delta_{x_{\perp} w_{\perp}}^{2} \delta_{x^{+} w^{+}}\right),
$$

see Eq. (49), and

$$
\begin{aligned}
\left(\left(U_{2}^{c b}\right)_{z t w}^{c a}\right)_{A_{+}, A_{-}=0}= & \frac{1}{2} N^{2} \delta^{a b}\left[\left(G_{z w}^{+0} G_{w t}^{+0}+G_{t w}^{+0} G_{w z}^{+0}\right)\right. \\
& +2\left(G_{z t}^{+0} G_{t w}^{+0}+G_{z t}^{+0} G_{w z}^{+0}\right. \\
& \left.\left.+G_{z w}^{+0} G_{t z}^{+0}+G_{t z}^{+0} G_{w t}^{+0}\right)\right] .
\end{aligned}
$$

Therefore, we obtain

$$
\begin{aligned}
-2 l\left(K_{x y 1}^{a b}\right)_{2}= & \frac{1}{2} g^{2} N \delta^{a b} \int \mathrm{d}^{4} z \mathrm{~d}^{4} t \mathrm{~d}^{4} w\left(\partial_{i z}^{2} G_{0++}^{t z}\right)\left(\delta_{x_{\perp} w_{\perp}}^{2} \delta_{x^{+} w^{+}}\right) \\
& \times\left(\delta_{y_{\perp} z_{\perp}}^{2} \delta_{y^{-} z^{-}}\right) \cdot\left[\left(G_{z w}^{+0} G_{w t}^{+0}+G_{t w}^{+0} G_{w z}^{+0}\right)\right. \\
& \left.+2\left(G_{z t}^{+0} G_{t w}^{+0}+G_{z t}^{+0} G_{w z}^{+0}+G_{z w}^{+0} G_{t z}^{+0}+G_{t z}^{+0} G_{w t}^{+0}\right)\right] .
\end{aligned}
$$




\section{$\left(N_{2}\right)_{a b}^{++}$term contribution}

The only contribution from the term $\left(N_{2}\right)_{a b}^{++}$in the vertex of interest comes from the expression

$$
-2 l\left(K_{x y 1}^{a b}\right)_{3}=\left[G_{0++} \frac{\delta^{2}\left(N_{2}\right)_{c c}^{++}}{\delta A_{+x}^{a} \delta A_{-y}^{b}}\right]_{A_{+}, A_{-}=0},
$$

which are determined by the following derivatives:

$$
\begin{aligned}
\frac{\delta^{2}\left(N_{2}\right)_{c c}^{++}}{\delta A_{+x}^{a} \delta A_{-y}^{b}}= & -2 g f_{c_{1} d c} \frac{\delta}{\delta A_{-y}^{b}}\left(\left(\partial_{-} v_{j}^{d c l}\right)-v_{j}^{d c l} \partial_{-}\right)_{t} \\
& \times \frac{\delta}{\delta A_{+x}^{a}}\left(\partial_{-z} \partial_{i z} \tilde{G}_{i j}^{c c_{1}}(z, t)\right) .
\end{aligned}
$$

Taking into account that

$$
\begin{aligned}
\frac{\delta}{\delta A_{+x}^{a}}\left(\partial_{-z} \partial_{i z} \tilde{G}_{i j}^{c c_{1}}(z, t)\right)= & -\int \mathrm{d}^{4} w\left(\partial_{-z} \partial_{i z} G_{0 i j_{1}}(z, w)\right) \\
& \times \frac{\delta\left(M_{1}(w)\right)_{j_{1} j_{2}}^{c c_{1}}}{\delta A_{+x}^{a}} G_{0 j_{2} j}(w, t),
\end{aligned}
$$

we collect all terms together obtaining

$$
\begin{aligned}
-2 l\left(K_{x y 1}^{a b}\right)_{3}= & 2 g f_{c_{1} d c} \int \mathrm{d}^{4} t \mathrm{~d}^{4} z \mathrm{~d}^{4} w G_{0++}(t, z)\left(\partial_{-z} \partial_{i z} G_{0 i j_{1}}(z, w)\right) \\
& \times \frac{\delta\left(M_{1 j_{1} j_{2}}^{c c l_{1}}\right)_{w}}{\delta A_{+x}^{a}}\left(\frac { \delta } { \delta A _ { - y } ^ { b } } \left(\left(\partial_{-} v_{j}^{d c l}\right)\right.\right. \\
& \left.\left.-v_{j}^{d c l} \partial_{-}\right)_{t} G_{0 j_{2} j}(w, t)\right) .
\end{aligned}
$$

Here we see from Eq. (58)

$\partial_{-} \rho_{i}^{a}=\frac{1}{N} \partial_{i} A_{-}^{a}$

that

$\frac{\delta}{\delta A_{-y}^{b}}\left(\left(\partial_{-} v_{j}^{d c l}\right)-v_{j}^{d c l} \partial_{-}\right)_{t}=-\delta^{d b} \delta_{y_{\perp} t_{\perp}}^{2}\left(\delta_{y^{-} t^{-}}-\tilde{G}_{t^{-} y^{-}}^{-0} \partial_{-t}\right) \partial_{j t}$.

\section{$\left(N_{3}\right)_{a b}^{++}$term contribution}

The $\left(N_{3}\right)_{a b}^{++}$term with required accuracy reads

$$
\begin{aligned}
\left(N_{3}\right)_{a b}^{++}= & \int \mathrm{d}^{4} z\left(\partial_{-x} \partial_{i x} G_{0 i j_{1}}(x, z)\right)\left(M_{1}(z)\right)_{j_{1} j_{2}}^{a d}\left(\partial_{-y} \partial_{j y} \tilde{G}_{j_{2} j}^{d b}(z, y)\right) \\
= & \int \mathrm{d}^{4} z\left(\partial_{-x} \partial_{i x} G_{0 i j_{1}}(x, z)\left(M_{1}(z)\right)_{j_{1} j_{2}}^{a b}\left(\partial_{-y} \partial_{j y} G_{0 j_{2} j}(z, y)\right)\right. \\
& -\int \mathrm{d}^{4} z \mathrm{~d}^{4} t\left(\partial_{-x} \partial_{i x} G_{0 i j_{1}}^{x z}\right)\left(M_{1_{j_{1} j_{2}}}^{a d}\right)_{z} G_{0 j_{2} j_{3}}^{z t}\left(M_{1 j_{3} j_{4}}^{d b}\right)_{t}\left(\partial_{-y} \partial_{j y} G_{0 j_{4 j} j}^{t y}\right),
\end{aligned}
$$

where we have the brief notation $G_{0 i j_{1}}(x, z) \rightarrow G_{0 i j_{1}}^{x z}$ in the last expression and only the $M_{1}$ term was preserved in comparison with Eq. (35). The $M_{2}$ does not contribute to the vertex of interest. Therefore, the first contribution to the kernel is given by the following expression:

$$
\begin{aligned}
& -2 l\left(K_{x y 1}^{a b}\right)_{4-a}=\left[G_{0++} \frac{\delta^{2}\left(N_{3}\right)_{c c}^{++}}{\delta A_{+x}^{a} \delta A_{-y}^{b}}\right]_{A_{+}, A_{-}=0} \\
& =-\left[G _ { 0 + + } \frac { \delta ^ { 2 } } { \delta A _ { + x } ^ { a } \delta A _ { - y } ^ { b } } \left(\int \mathrm{d}^{4} z \mathrm{~d}^{4} t\left(\partial_{-w} \partial_{i w} G_{0 i j_{1}}^{w z}\right)\left(M_{1 j_{1} j_{2}}^{c d}\right)_{z}\right.\right. \\
& \left.\left.\times G_{0 j_{2} j_{3}}^{z t}\left(M_{1 j_{3} j_{4}}^{d c}\right)_{t}\left(\partial_{-s} \partial_{j s} G_{0 j_{4} j}^{t s}\right)\right)\right]_{A_{+}, A_{-}=0} \\
& =-2 \int \mathrm{d}^{4} w \mathrm{~d}^{4} s \mathrm{~d}^{4} z \mathrm{~d}^{4} t\left(G_{0++}^{s w}\left(\partial_{-w} \partial_{i w} G_{0 i j_{1}}^{w z}\right) \frac{\delta\left(M_{1 j_{1} j_{2}}^{c d}\right)_{z}}{\delta A_{+x}^{a}} G_{0 j_{2} j_{3}}^{z t}\right. \\
& \left.\times \frac{\left(M_{1 j_{3} j_{4}}^{d c}\right)_{t}}{\delta A_{-y}^{b}}\left(\partial_{-s} \partial_{j s} G_{0 j_{4} j}^{t s}\right)\right)_{A_{+}, A_{-}=0} .
\end{aligned}
$$

The second contribution of this term to the kernel is given by

$$
\begin{aligned}
& -2 l\left(K_{x y 1}^{a b}\right)_{4-b} \\
& =-\left[G_{0++} \frac{\delta\left(N_{3}\right)_{c d}^{++}}{\delta A_{-y}^{b}} G_{0++} \frac{\delta\left(N_{3}\right)_{d c}^{++}}{\delta A_{+x}^{a}}\right]_{A_{+}, A_{-}=0} .
\end{aligned}
$$

Inserting the leading contribution from Eq. (B.18) in Eq. (B.20) we obtain

$$
\begin{aligned}
& -2 l\left(K_{x y 1}^{a b}\right)_{4-b}=-\int \mathrm{d}^{4} w \mathrm{~d}^{4} s \mathrm{~d}^{4} z \mathrm{~d}^{4} t \int \mathrm{d}^{4} w_{1} \mathrm{~d}^{4} t_{1} \\
& \times\left(G_{0++}^{s w}\left(\partial_{-w} \partial_{i w} G_{0 i j_{1}}^{w w_{1}}\right)\left(\frac{\delta}{\delta A_{+x}^{a}}\left(M_{1 j_{1} j_{2}}^{c d}\right)_{w_{1}}\right)\right. \\
& \times\left(\partial_{-z} \partial_{j z} G_{0 j_{2} j}^{w_{1} z}\right) G_{0++}^{z t}\left(\partial_{-t} \partial_{l t} G_{0 l l_{1}}^{t t_{1}}\right) \\
& \left.\times\left(\frac{\delta}{\delta A_{-y}^{b}}\left(M_{1 l_{1} l_{2}}^{d c}\right)_{t_{1}}\right)\left(\partial_{-s} \partial_{m s} G_{0 l_{2} m}^{t_{1} s}\right)\right)_{A_{+}, A_{-}=0}
\end{aligned}
$$

We introduce now an additional operator:

$$
\begin{aligned}
\hat{G}_{i j}(x, y)= & G_{0 i j}(x, y)+\int \mathrm{d}^{4} z \mathrm{~d}^{4} t\left(\partial_{-z} \partial_{k z} G_{0 i k}(x, z)\right) \\
& \times G_{0++}(z, t)\left(\partial_{-t} \partial_{l t} G_{0 l j}(t, y)\right) \\
= & -\delta_{i j} \int \frac{\mathrm{d}^{4} p}{(2 \pi)^{4}} \frac{e^{-\imath p(x-y)}}{p^{2}}
\end{aligned}
$$

and rewrite the sum

$$
\left(K_{x y 1}^{a b}\right)_{1,4}=\left(K_{x y 1}^{a b}\right)_{1}+\left(K_{x y 1}^{a b}\right)_{4-a}+\left(K_{x y 1}^{a b}\right)_{4-b}
$$

as

$$
\begin{aligned}
-2 \imath\left(K_{x y 1}^{a b}\right)_{1,4}= & -\int \mathrm{d}^{4} z \mathrm{~d}^{4} t \hat{G}_{i j}^{z t}\left(\frac{\delta}{\delta A_{+x}^{a}}\left(M_{1 j j_{1}}^{c d}\right)_{t}\right) \\
& \times \hat{G}_{j_{i} j_{2}}^{t z}\left(\frac{\delta}{\delta A_{-y}^{b}}\left(M_{1 j_{2} i}^{d c}\right)_{z}\right) .
\end{aligned}
$$


The functional derivatives in the above expression are given by

$\frac{\delta}{\delta A_{+x}^{a}}\left(M_{1 j j_{1}}^{c d}\right)_{t}=2 g \delta_{j j_{1}} \delta_{t_{\perp} x_{\perp}}^{2} \delta_{t^{+} x^{+}} f_{c a d} \partial_{-t}$

and

$\frac{\delta}{\delta A_{-y}^{b}}\left(M_{1 j_{2} i}^{d c}\right)_{z}=2 g f_{d b c} \tilde{G}_{z^{-} y^{-}}^{-0} \delta_{z_{\perp} y_{\perp}}^{2}\left(\delta_{j_{2} i}\left(\partial_{\perp z}\right)^{2}-\partial_{j_{2} z} \partial_{i z}\right)$.

$\left(N_{1}\right)_{a b}^{++}$and $\left(N_{3}\right)_{a b}^{++}$terms contribution

For the non-diagonal contribution from the $\left(N_{1}\right)_{a b}^{++}$and $\left(N_{3}\right)_{a b}^{++}$terms we have

$$
-2 \imath\left(K_{x y 1}^{a b}\right)_{5}=-\left[G_{0++} \frac{\delta\left(N_{1}\right)_{c d}^{++}}{\delta A_{-y}^{b}} G_{0++} \frac{\delta\left(N_{3}\right)_{d c}^{++}}{\delta A_{+x}^{a}}\right]_{A_{+}, A_{-}=0} .
$$

Using results from the previous chapters, we obtain

$$
\begin{aligned}
& -2 l\left(K_{x y 1}^{a b}\right)_{5}=-\frac{g}{N} \int \mathrm{d}^{4} w \mathrm{~d}^{4} s \mathrm{~d}^{4} z \mathrm{~d}^{4} t \mathrm{~d}^{4} w_{1} \\
& \times\left(G_{0++}^{z t}\left(\left(U_{1}^{c b}\right)_{t w}^{d}\right)_{A_{+}, A_{-}=0}\left(\delta_{t_{\perp} y_{\perp}}^{a} \delta_{t^{-} y^{-}} \partial_{i t}^{2}\right)\right. \\
& \left.\cdot G_{0++}^{w s}\left(\partial_{-s} \partial_{i s} G_{0 i j_{1}}^{s w_{1}}\right)\left(\frac{\delta}{\delta A_{+x}^{a}}\left(M_{1 j_{1} j_{2}}^{d c}\right)_{w_{1}}\right)\left(\partial_{-z} \partial_{j z} G_{0 j_{2} j}^{w_{1} z}\right)\right) .
\end{aligned}
$$

Here we have

$\left(\left(U_{1}^{c b}\right)_{t w}^{d}\right)_{A_{+}, A_{-}=0}=\frac{1}{2} N f_{c d b}\left(G_{t w}^{+0}-G_{w t}^{+0}\right) ;$

see Eq. (26). Now we introduce new operators:

$$
\begin{aligned}
\hat{G}_{+j}(x, y) & =\int \mathrm{d}^{4} z G_{0++}(x, z)\left(\partial_{-z} \partial_{i z} G_{0 i j}(z, y)\right) \\
& =-\int \frac{\mathrm{d}^{4} p}{(2 \pi)^{4}} \frac{e^{-\imath p(x-y)}}{p^{2}} \frac{p_{j}}{p_{-}}
\end{aligned}
$$

and

$$
\begin{aligned}
\hat{G}_{j+}(x, y) & =\int \mathrm{d}^{4} z\left(\partial_{-z} \partial_{i z} G_{0 i j}(x, z)\right) G_{0++}(z, y) \\
& =\hat{G}_{+j}(y, x) .
\end{aligned}
$$

Finally, with the help of these operators, we rewrite Eq. (B.28) as

$$
\begin{aligned}
-2 l\left(K_{x y 1}^{a b}\right)_{5}= & -\frac{1}{2} g f_{c d b} \int \mathrm{d}^{4} w \mathrm{~d}^{4} t \mathrm{~d}^{4} w_{1}\left(\left(G_{t w}^{+0}-G_{w t}^{+0}\right)\left(\delta_{t_{\perp} y_{\perp}}^{a} \delta_{t^{-} y^{-}}\right) .\right. \\
& \left.\cdot \hat{G}_{+j_{1}}^{w w_{1}}\left(\frac{\delta}{\delta A_{+x}^{a}}\left(M_{1 j_{1} j_{2}}^{d c}\right)_{w_{1}}\right)\left(\partial_{i t}^{2} \hat{G}_{j_{2}+}^{w_{1} t}\right)\right) .
\end{aligned}
$$

\section{$\left(N_{2}\right)_{a b}^{++}$and $\left(N_{3}\right)_{a b}^{++}$terms contribution}

For the non-diagonal contribution from the terms $\left(N_{2}\right)_{a b}^{++}$ and $\left(N_{3}\right)_{a b}^{++}$we have

$$
-2 l\left(K_{x y 1}^{a b}\right)_{6}=-\left[G_{0++} \frac{\delta\left(N_{2}\right)_{c d}^{++}}{\delta A_{-y}^{b}} G_{0++} \frac{\delta\left(N_{3}\right)_{d c}^{++}}{\delta A_{+x}^{a}}\right]_{A_{+}, A_{-}=0} .
$$

Using the results of previous calculations we write

$$
\begin{aligned}
\frac{\delta\left(\left(N_{2}\right)_{c d}^{++}\right)_{t w}}{\delta A_{-y}^{b}}= & -2 g f_{c_{1} d_{1} d}\left(\frac{\delta}{\delta A_{-y}^{b}}\left(\left(\partial_{-} v_{j}^{d_{1} c l}\right)-v_{j}^{d_{1} c l} \partial_{-}\right)_{w}\right) \\
& \times\left(\partial_{-t} \partial_{i t} \tilde{G}_{i j}^{c c_{1}}(t, w)\right)
\end{aligned}
$$

and

$\frac{\delta\left(N_{3}\right)_{d c}^{++}}{\delta A_{+x}^{a}}=\int \mathrm{d}^{4} w_{1}\left(\partial_{-s} \partial_{i s} G_{0 i j_{1}}^{s w_{1}}\right)\left(\frac{\delta}{\delta A_{+x}^{a}}\left(M_{1 j_{1} j_{2}}^{d c}\right)_{w_{1}}\right)\left(\partial_{-z} \partial_{j z} G_{0 j_{2} j}^{w_{1} z}\right)$.

Therefore, we have for the contribution of Eq. (B.33)

$$
\begin{aligned}
& -2 l\left(K_{x y 1}^{a b}\right)_{6}=2 g f_{c d_{1} d} \int \mathrm{d}^{4} z \mathrm{~d}^{4} t \mathrm{~d}^{4} w \mathrm{~d}^{4} s \mathrm{~d}^{4} w_{1} \\
& \times\left(G_{0++}^{z t}\left(\frac{\delta}{\delta A_{-y}^{b}}\left(\left(\partial_{-} v_{j}^{d_{1} c l}\right)-v_{j}^{d_{1} c l} \partial_{-}\right)_{w}\right)\right. \\
& \left(\partial_{-t} \partial_{i t} G_{0 i j}^{t w}\right) G_{0++}^{w s}\left(\partial_{-s} \partial_{i s} G_{0 i j_{1}}^{s w_{1}}\right) \\
& \left.\left(\frac{\delta}{\delta A_{+x}^{a}}\left(M_{1 j_{1} j_{2}}^{d c}\right)_{w_{1}}\right)\left(\partial_{-z} \partial_{j z} G_{0 j_{2} j}^{w_{1} z}\right)\right) \\
& =2 g f_{c d_{1} d} \int \mathrm{d}^{4} w \mathrm{~d}^{4} w_{1}\left(\frac{\delta}{\delta A_{-y}^{b}}\left(\left(\partial_{-} v_{j}^{d_{1} c l}\right)-v_{j}^{d_{1} c l} \partial_{-}\right)_{w}\right. \\
& \left.\quad \times\left(\hat{G}_{j_{2} j}^{w_{1} w}-G_{0 j_{2} j}^{w_{1} w}\right)\right) \hat{G}_{+j_{1}}^{w w_{1}}\left(\frac{\delta}{\delta A_{+x}^{a}}\left(M_{1 j_{1} j_{2}}^{c d}\right)_{w_{1}}\right) .
\end{aligned}
$$

Now the sum

$\left(K_{x y 1}^{a b}\right)_{6,3}=\left(K_{x y 1}^{a b}\right)_{6}+\left(K_{x y 1}^{a b}\right)_{3}$

can be written as

$$
\begin{aligned}
-2 l\left(K_{x y 1}^{a b}\right)_{6,3} \\
=2 g f_{c d_{1} d} \int \mathrm{d}^{4} w \mathrm{~d}^{4} w_{1} \hat{G}_{+j_{1}}^{w w_{1}}\left(\frac{\delta}{\delta A_{+x}^{a}}\left(M_{1 j_{1} j_{2}}^{d c}\right)_{w_{1}}\right) . \\
\cdot\left(\left(\frac{\delta}{\delta A_{-y}^{b}}\left(\left(\partial_{-} v_{j}^{d_{1} c l}\right)-v_{j}^{d_{1} c l} \partial_{-}\right)_{w}\right) \hat{G}_{j_{2} j}^{w_{1} w}\right),
\end{aligned}
$$

with the derivatives in the expression given by Eqs. (B.17) and (B.25). 


\section{Final expression for the kernel}

Taking all contributions together we obtain the following expression for the kernel to required order:

$$
\begin{aligned}
-2 & K_{x y 1}^{a b}=\frac{1}{2} g^{2} N \delta^{a b} \int \mathrm{d}^{4} z \mathrm{~d}^{4} t \mathrm{~d}^{4} w\left(\partial_{i z}^{2} G_{0++}^{t z}\right) \\
& \times\left(\delta_{x_{\perp} w_{\perp}}^{2} \delta_{x^{+} w^{+}}\right)\left(\delta_{y_{\perp} z_{\perp}}^{2} \delta_{y^{-} z^{-}}\right) \cdot\left[\left(G_{z w}^{+0} G_{w t}^{+0}+G_{t w}^{+0} G_{w z}^{+0}\right)\right. \\
& \left.+2\left(G_{z t}^{+0} G_{t w}^{+0}+G_{z t}^{+0} G_{w z}^{+0}+G_{z w}^{+0} G_{t z}^{+0}+G_{t z}^{+0} G_{w t}^{+0}\right)\right] \\
& -\int \mathrm{d}^{4} z \mathrm{~d}^{4} t \hat{G}_{i j}^{z t}\left(\frac{\delta}{\delta A_{+x}^{a}}\left(M_{1 j_{1}}^{c d}\right)_{t}\right) \hat{G}_{j_{i} j_{2}}^{t z}\left(\frac{\delta}{\delta A_{-y}^{b}}\left(M_{1 j_{2} i}^{d c}\right)_{z}\right) \\
& -\frac{1}{2} g f_{c d b} \int \mathrm{d}^{4} w \mathrm{~d}^{4} t \mathrm{~d}^{4} w_{1}\left(G_{t w}^{+0}-G_{w t}^{+0}\right)\left(\delta_{t_{\perp} y_{\perp}}^{2} \delta_{t^{-} y^{-}}\right) \hat{G}_{+j_{1}}^{w w_{1}} \\
& \times\left(\frac{\delta}{\delta A_{+x}^{a}}\left(M_{1 j_{1} j_{2}}^{d c}\right)_{w_{1}}\right)\left(\partial_{i t}^{2} \hat{G}_{j_{2}+}^{w_{1} t}\right) \\
+ & 2 g f_{c d_{1} d} \int \mathrm{d}^{4} w \mathrm{~d}^{4} w_{1} \hat{G}_{+j_{1}}^{w w_{1}}\left(\frac{\delta}{\delta A_{+x}^{a}}\left(M_{1 j_{1} j_{2}}^{d c}\right)_{w_{1}}\right) \\
\times & \left(\frac{\delta}{\delta A_{-y}^{b}}\left(\left(\partial_{-} v_{j}^{d_{1} c l}\right)-v_{j}^{d_{1} c l} \partial_{-}\right)_{w}\right) \hat{G}_{j_{2} j}^{w_{1} w},
\end{aligned}
$$

where the expressions for all functional derivatives are determined above.

\section{Appendix C: Calculation of the final answer for $K_{x y 1}^{a b}$ effective kernel}

Below we present the final answer for the vertex of interest after the calculations of integrals in Eq. (B.39). First of all, we calculate the integrals from Eq. (B.39) which give nonzero contributions, we consider the other integrals from Eq. (B.39) in the end of the appendix. We also recall that in the framework of the approach we consider the cluster of the particles with ${ }^{5} p_{-}>0$, therefore we have

$$
\frac{1}{p^{2}} \rightarrow \frac{1}{2 p_{-}\left(p_{+}-\frac{p_{\perp}^{2}}{2 p_{-}}+\imath \varepsilon\right)}, \varepsilon>0,
$$

which determines the form of the integration contours in the $p_{+}$integrals.

Non-zero contribution: first integral of Eq. (73)

This contribution reads

$$
\begin{aligned}
I_{1}= & \frac{1}{2} g^{2} N \delta^{a b} \int \mathrm{d}^{4} z \mathrm{~d}^{4} t \mathrm{~d}^{4} w\left(\partial_{i z}^{2} G_{0++}^{t z}\right)\left(\delta_{x_{\perp} w_{\perp}}^{2} \delta_{x^{+} w^{+}}\right) \\
& \times\left(\delta_{y_{\perp} z_{\perp}}^{2} \delta_{y^{-} z^{-}}\right)\left(G_{t w}^{+0} G_{w z}^{+0}-2\left(G_{z w}^{+0} G_{t z}^{+0}+G_{t z}^{+0} G_{w t}^{+0}\right)\right)
\end{aligned}
$$

\footnotetext{
5 The full answer Eq. (B.39) is symmetrical with respect to sign of $p_{-}$ momentum and the final answer does not depend on it.
}

or, using the results of Appendix A and Eq. (47), we write it as

$$
\begin{aligned}
I_{1}= & g^{2} N \delta^{a b} \partial_{i x}^{2}\left(\delta_{x \perp y \perp}^{2} \int \mathrm{d} t^{+} \theta\left(t^{+}-x^{+}\right) \int \mathrm{d} z^{+} \theta\left(x^{+}-z^{+}\right)\right. \\
& \left.\times \int \mathrm{d} p_{-} \int \mathrm{d} p_{+} \int \frac{\mathrm{d}^{2} p_{\perp}}{(2 \pi)^{4}} \frac{p_{+}}{p^{2} p_{-}} e^{-\imath p_{+}\left(t^{+}-z^{+}\right)}\right) .
\end{aligned}
$$

The integrals are found after the regularization:

$$
\begin{aligned}
& \int \mathrm{d} t^{+} \theta\left(t^{+}-x^{+}\right) e^{-\imath p_{+} t^{+}}=-\frac{\imath}{p_{+}-\imath \varepsilon} e^{-\imath p_{+} x^{+}} \\
& \text {and } \\
& \int \mathrm{d} z^{+} \theta\left(x^{+}-z^{+}\right) e^{\imath p_{+} z^{+}}=\frac{l}{p_{+}-\imath \varepsilon} e^{\imath p_{+} x^{+}}
\end{aligned}
$$

Inserting these expressions in Eq. (C.3) we obtain

$$
\begin{aligned}
I_{1}= & -g^{2} N \delta^{a b} \partial_{i x}^{2}\left(\delta_{x_{\perp} y_{\perp}}^{2} \int \frac{\mathrm{d} p_{-}}{2 p_{-}^{2}}\right. \\
& \left.\times \int \frac{\mathrm{d}^{2} p_{\perp}}{(2 \pi)^{4}} \int \mathrm{d} p_{+} \frac{1}{p_{+}-\frac{p_{\perp}^{2}}{2 p_{-}}+\imath \varepsilon} \frac{1}{p_{+}-\imath \varepsilon}\right),
\end{aligned}
$$

which after the integration on $p_{+}$gives finally

$$
I_{1}=\imath \frac{g^{2} N}{(2 \pi)^{3}} \delta^{a b} \partial_{i x}^{2}\left(\int \frac{\mathrm{d} p_{-}}{p_{-}} \int \frac{\mathrm{d}^{2} p_{\perp}}{p_{\perp}^{2}} \int \frac{\mathrm{d}^{2} k_{\perp}}{(2 \pi)^{2}} e^{-l k_{\perp}\left(x_{\perp}-y_{\perp}\right)}\right) .
$$

\section{Non-zero contribution: second integral of Eq. (73)}

This contribution reads

$$
\begin{aligned}
I_{2}= & \frac{1}{2} g f_{c d b} \int \mathrm{d}^{4} w \mathrm{~d}^{4} t \mathrm{~d}^{4} w_{1} G_{w t}^{+0}\left(\delta_{t_{\perp} y_{\perp}}^{2} \delta_{t^{-} y^{-}}\right) \\
& \times \hat{G}_{+j_{1}}^{w w_{1}}\left(\frac{\delta}{\delta A_{+x}^{a}}\left(M_{1 j_{1} j_{2}}^{d c}\right)_{w_{1}}\right)\left(\partial_{i t}^{2} \hat{G}_{j_{2}+}^{w_{1} t}\right) .
\end{aligned}
$$

Here we have

$\frac{\delta}{\delta A_{+x}^{a}}\left(M_{1 j_{1} j_{2}}^{d c}\right)_{w_{1}}=2 g \delta_{j_{1} j_{2}} \delta_{w_{1 \perp} x_{\perp}}^{2} \delta_{w_{1}^{+} x^{+}} f_{d a c} \partial_{-w_{1}}$

and

$G_{w t}^{+0}=\theta\left(w^{+}-t^{+}\right) \delta_{w_{\perp} t_{\perp}}^{2} \delta_{w^{-} t^{-}}$

see Eq. (B.25) and Appendix A. Inserting these expressions in Eq. (C.8) we obtain for the first contribution

$$
\begin{aligned}
I_{2}= & g^{2} N \delta^{a b} \int \mathrm{d} w^{+} \theta\left(w^{+}-t^{+}\right) \int \mathrm{d} t^{+} \int \mathrm{d} w_{1}^{+} \delta_{w_{1}^{+} x^{+}} \\
& \times \int \mathrm{d} w^{-} \delta_{w^{-} t^{-}} \int \mathrm{d} t^{-} \delta_{t^{-} y^{-}}
\end{aligned}
$$




$$
\begin{aligned}
& \int \mathrm{d} w_{1}^{-} \int \mathrm{d}^{2} w_{\perp} \delta_{w_{\perp} t_{\perp}}^{2} \int \mathrm{d}^{2} t_{\perp} \delta_{t_{\perp} y_{\perp}}^{2} \\
& \times \int \mathrm{d}^{2} w_{1 \perp} \delta_{w_{1 \perp} x_{\perp}}^{2} \hat{G}_{+j}^{w w_{1}}\left(\partial_{-w_{1}} \partial_{i t}^{2} \hat{G}_{j+}^{w_{1} t}\right) .
\end{aligned}
$$

The propagator in Eq. (C.11) is defined above, see Eq. (B.30); it is

$\hat{G}_{+j}^{w w_{1}}=-\int \frac{\mathrm{d}^{4} p}{(2 \pi)^{4}} \frac{e^{-l p\left(w-w_{1}\right)}}{p^{2}} \frac{p_{j}}{p_{-}}$,

and correspondingly

$\partial_{-w_{1}} \partial_{i t}^{2} \hat{G}_{j+}^{w_{1} t}=-\imath \partial_{i t}^{2} \int \frac{\mathrm{d}^{4} k}{(2 \pi)^{4}} e^{\imath k\left(w_{1}-t\right)} \frac{k_{j}}{k^{2}}$.

Now, performing the integration on the delta-functions, we obtain

$$
\begin{aligned}
& I_{2}=l g^{2} N \delta^{a b} \partial_{i x}^{2}\left(\int \mathrm{d} t^{+} \int \mathrm{d} w^{+} \theta\left(w^{+}-t^{+}\right)\right. \\
& \times \int \mathrm{d} w_{1}^{-} e^{\imath w_{1}^{-}\left(p_{-}+k_{-}\right)} \int \frac{\mathrm{d}^{4} p}{(2 \pi)^{4}} \\
& \int \frac{\mathrm{d}^{4} k}{(2 \pi)^{4}} \frac{p_{j}}{p_{-} p^{2}} \frac{k_{j}}{k^{2}} e^{-l y^{-}\left(p_{-}+k_{-}\right)} e^{-l\left(x_{i}-y_{i}\right)\left(p_{i}+k_{i}\right)} \\
& \left.\times e^{-l p_{+}\left(w^{+}-x^{+}\right)} e^{\imath k_{+}\left(x^{+}-t^{+}\right)}\right) .
\end{aligned}
$$

The integral on the variable $w_{1}^{-}$leads to

$\int \mathrm{d} w_{1}^{-} e^{\imath w_{1}^{-}\left(p_{-}+k_{-}\right)}=2 \pi \delta_{p_{-}-k_{-}}$,

and integration on the variable $w^{+}$gives

$$
\begin{aligned}
\int \mathrm{d} w^{+} \theta\left(w^{+}-t^{+}\right) e^{-l p_{+} w^{+}} & =\int_{t^{+}}^{\infty} \mathrm{d} w^{+} e^{-l w^{+}\left(p_{+}-\imath \varepsilon\right)} \\
& =-\frac{l}{p_{+}-\imath \varepsilon} e^{-l p_{+} t^{+}} .
\end{aligned}
$$

Therefore, we obtain for Eq. (C.15)

$$
\begin{aligned}
I_{2}= & -2 \pi g^{2} N \delta^{a b} \partial_{i x}^{2}\left(\int \mathrm{d} k_{+} \int \mathrm{d} t^{+} e^{-\imath t^{+}\left(p_{+}+k_{+}\right)} \int \frac{\mathrm{d} p_{-}}{p_{-}}\right. \\
& \times \int \mathrm{d} p_{+} \int \frac{\mathrm{d}^{2} p_{\perp}}{(2 \pi)^{4}} \int \frac{\mathrm{d}^{2} k_{\perp}}{(2 \pi)^{4}} \frac{1}{p_{+}-\imath \varepsilon} \\
& \times \frac{p_{j}}{2 p_{-}\left(p_{+}-\frac{p_{\perp}^{2}}{2 p_{-}}+\imath \varepsilon\right)} \frac{k_{j}}{2 p_{-}\left(k_{+}+\frac{k_{\perp}^{2}}{2 p_{-}}-\imath \varepsilon\right)} \\
& \left.\times e^{-l\left(p_{i}-k_{i}\right)\left(x_{i}-y_{i}\right)} e^{-\imath x^{+}\left(k_{+}-p_{+}\right)}\right) .
\end{aligned}
$$

Integrating now on the variables $t^{+}$and $k_{+}$we have

$$
\begin{aligned}
I_{2}= & (2 \pi)^{2} g^{2} N \delta^{a b} \partial_{i x}^{2}\left(\int \frac{\mathrm{d} p_{-}}{p_{-}} \int \frac{\mathrm{d}^{2} p_{\perp}}{(2 \pi)^{4}}\right. \\
& \times \int \frac{\mathrm{d}^{2} k_{\perp}}{(2 \pi)^{4}}\left(p_{j} k_{j}\right) e^{-l\left(p_{i}+k_{i}\right)\left(x_{i}-y_{i}\right)} \\
& \left.\times \int \frac{\mathrm{d} p_{+}}{4 p_{-}^{2}} \frac{1}{\left(p_{+}-\frac{k_{\perp}^{2}}{2 p_{-}}+\imath \varepsilon\right)} \frac{1}{\left(p_{+}-\frac{p_{\perp}^{2}}{2 p_{-}}+\imath \varepsilon\right)} \frac{1}{\left(p_{+}-\imath \varepsilon\right)}\right),
\end{aligned}
$$

and performing the $p_{+}$integration we obtain

$$
\begin{aligned}
I_{2}= & \frac{l g^{2} N}{2 \pi} \delta^{a b} \partial_{i x}^{2}\left(\int \frac{\mathrm{d} p_{-}}{p_{-}} \int \frac{\mathrm{d}^{2} p_{\perp}}{(2 \pi)^{2}}\right. \\
& \left.\times \int \frac{\mathrm{d}^{2} k_{\perp}}{(2 \pi)^{2}} \frac{p_{j} k_{j}}{p_{\perp}^{2} k_{\perp}^{2}} e^{-l\left(p_{i}+k_{i}\right)\left(x_{i}-y_{i}\right)}\right) .
\end{aligned}
$$

Performing the variable change $p_{\perp}+k_{\perp} \rightarrow k_{\perp}$ we rewrite the integral in the more familiar form

$$
\begin{aligned}
I_{2}= & \iota \delta^{a b} \frac{g^{2} N}{4 \pi} \partial_{i x}^{2}\left(\int \frac{\mathrm{d} p_{-}}{p_{-}} \int \frac{\mathrm{d}^{2} p_{\perp}}{(2 \pi)^{2}}\right. \\
& \left.\times \int \frac{\mathrm{d}^{2} k_{\perp}}{(2 \pi)^{2}} \frac{k_{\perp}^{2}}{p_{\perp}^{2}\left(p_{\perp}-k_{\perp}\right)^{2}} e^{-l k_{i}\left(x_{i}-y_{i}\right)}\right) \\
& -\imath \delta^{a b} \frac{g^{2} N}{(2 \pi)^{3}} \partial_{i x}^{2}\left(\int \frac{\mathrm{d} p_{-}}{p_{-}} \int \frac{\mathrm{d}^{2} p_{\perp}}{p_{\perp}^{2}} \int \frac{\mathrm{d}^{2} k_{\perp}}{(2 \pi)^{2}} e^{-l k_{i}\left(x_{i}-y_{i}\right)}\right) .
\end{aligned}
$$

\section{Zero contributions in Eq. (B.39)}

First of all, consider the term $G_{z w}^{+0} G_{w t}^{+0}$ in the first integral term in Eq. (B.39). With the help of Appendix A and performing the calculation above we are left with the following expression for this term:

$$
\begin{aligned}
& \theta\left(z^{+}-t^{+}\right) \int \mathrm{d} p^{+} \frac{e^{l p_{+}\left(z^{+}-t^{+}\right)}}{p_{+}-\frac{p_{\perp}^{2}}{2 p_{-}}+\imath \varepsilon} \propto \theta\left(z^{+}-t^{+}\right) \theta\left(t^{+}-z^{+}\right) \\
& \quad=0
\end{aligned}
$$

see the definition of Eq. (C.1). The same holds for the $G_{z t}^{+0} G_{t w}^{+0}$ and $G_{z t}^{+0} G_{w z}^{+0}$ terms and for the second and fourth terms in Eq. (B.39). Two last terms in the first term in Eq. (B.39) are equal to zero after the integration on the transverse momentum in the corresponding integrals.

\section{References}

1. L.N. Lipatov, Nucl. Phys. B 452, 369 (1995)

2. L.N. Lipatov, Phys. Rep. 286, 131 (1997)

3. L.N. Lipatov, Subnucl. Ser. 49, 131 (2013)

4. L.N. Lipatov, Int. J. Mod. Phys. Conf. Ser. 39, 1560082 (2015)

5. L.N. Lipatov, Int. J. Mod. Phys. A 31(28/29), 1645011 (2016) 
6. L.N. Lipatov, EPJ Web Conf. 125, 01010 (2016)

7. S. Bondarenko, L. Lipatov, A. Prygarin, Eur. Phys. J. C 77(8), 527 (2017)

8. V.N. Gribov, Sov. Phys. JETP 26, 414 (1968)

9. L.N. Lipatov, Nucl. Phys. Proc. Suppl. 99A, 175 (2001)

10. M.A. Braun, M.I. Vyazovsky, Eur. Phys. J. C 51, 103 (2007)

11. M.A. Braun, M.Y. Salykin, M.I. Vyazovsky, Eur. Phys. J. C 65, $385(2010)$

12. M.A. Braun, L.N. Lipatov, M.Y. Salykin, M.I. Vyazovsky, Eur. Phys. J. C 71, 1639 (2011)

13. M.A. Braun, M.Y. Salykin, M.I. Vyazovsky, Eur. Phys. J. C 72, 1864 (2012)

14. M. Hentschinski, A.S. Vera, Phys. Rev. D 85, 056006 (2012)

15. M.A. Braun, M.Y. Salykin, S.S. Pozdnyakov, M.I. Vyazovsky, Eur. Phys. J. C 72, 2223 (2012)

16. J. Bartels, L.N. Lipatov, G.P. Vacca, Phys. Rev. D 86, 105045 (2012)

17. M.A. Braun, S.S. Pozdnyakov, M.Y. Salykin, M.I. Vyazovsky, Eur. Phys. J. C 73(9), 2572 (2013)

18. G. Chachamis, M. Hentschinski, J.D.M. Martnez, A.S. Vera, Phys. Part. Nucl. 45(4), 788 (2014)

19. M.A. Braun, Eur. Phys. J. C 75(7), 298 (2015)

20. M.A. Braun, M.I. Vyazovsky, Phys. Rev. D 93(6), 065026 (2016)

21. M.A. Braun, Eur. Phys. J. C 77(5), 279 (2017)

22. M.A. Braun, M.Y. Salykin, Eur. Phys. J. C 77(7), 498 (2017)

23. J. Bartels, Nucl. Phys. B 175, 365 (1980)

24. J. Kwiecinski, M. Praszalowicz, Phys. Lett. B 94, 413 (1980)

25. J. Bartels, V.S. Fadin, L.N. Lipatov, G.P. Vacca, Nucl. Phys. B 867, 827 (2013)

26. L.V. Gribov, E.M. Levin, M.G. Ryskin, Phys. Rep. 100, 1 (1983)

27. I. Balitsky, Nucl. Phys. B 463, 99 (1996)

28. Y.V. Kovchegov, Phys. Rev. D 60, 034008 (1999)

29. Y.V. Kovchegov, Phys. Rev. D 61, 074018 (2000)

30. I. Balitsky, Phys. Rev. D 60, 014020 (1999)

31. I. Balitsky, in At the frontier of particle physics, vol. 2*, ed. by *M. Shifman (1999), pp 1237-1342

32. I.I.Balitsky, A.V.Belitsky, Nucl. Phys. B 629, 290 (2002)

33. L.N. Lipatov, Sov. J. Nucl. Phys. 23, 338 (1976)

34. L.N. Lipatov, Yad. Fiz. 23, 642 (1976)

35. E.A. Kuraev, L.N. Lipatov, V.S. Fadin, Sov. Phys. JETP 45, 199 (1977)

36. E.A. Kuraev, L.N. Lipatov, V.S. Fadin, Zh. Eksp. Teor. Fiz. 72, 377 (1977)

37. I.I. Balitsky, L.N. Lipatov, Sov. J. Nucl. Phys. 28, 822 (1978)

38. I.I. Balitsky, L.N. Lipatov, Yad. Fiz. 28, 1597 (1978)

39. J. Bartels, Z. Phys. C 60, 471 (1993)

40. J. Bartels, M. Wusthoff, Z. Phys. C 66, 157 (1995)

41. J. Bartels, C. Ewerz, JHEP 9909, 026 (1999)

42. L. McLerran, R. Venugopalan, Phys. Rev. D 49, 2233 (1994)

43. L. McLerran, R. Venugopalan, Phys. Rev. D 49, 3352 (1994)

44. J. Jalilian-Marian, A. Kovner, L. McLerran, H. Weigert, Phys. Rev. D 55, 5414 (1997)
45. J. Jalilian-Marian, A. Kovner, A. Leonidov, H. Weigert, Nucl. Phys. B 504, 415 (1997)

46. J. Jalilian-Marian, A. Kovner, A. Leonidov, H. Weigert, Phys. Rev. D 59, 014014 (1998)

47. E. Iancu, A. Leonidov, L.D. McLerran, Nucl. Phys. A 692, 583 (2001)

48. E. Iancu, A. Leonidov, L.D. McLerran, Phys. Lett. B 510, 133 (2001)

49. E. Ferreiro, E. Iancu, A. Leonidov, L. McLerran, Nucl. Phys. A 703, 489 (2002)

50. I. Balitsky, Phys. Rev. D 72, 074027 (2005)

51. Y. Hatta, Nucl. Phys. A 768, 222 (2006)

52. Y. Hatta, E. Iancu, L. McLerran, A. Stasto, D.N. Triantafyllopoulos, Nucl. Phys. A 764, 423 (2006)

53. Y. Hatta, Nucl. Phys. A 781, 104 (2007)

54. A. Basseto, G. Nardelli, R. Soldati, Yang Mills theories in algebraic non-covariant gauges (World Scientific, Singapore, 1991)

55. S.J. Brodsky, H.C. Pauli, S.S. Pinsky, Phys. Rep. 301, 299 (1998)

56. A. Ayala, J. Jalilian-Marian, L.D. McLerran, R. Venugopalan, Phys. Rev. D 52, 2935 (1995)

57. M.A. Braun, Phys. Lett. B 483, 115 (2000)

58. M.A. Braun, Eur. Phys. J. C 33, 113 (2004)

59. S. Bondarenko, M.A. Braun, Nucl. Phys. A 799, 151 (2008)

60. S. Bondarenko, Nucl. Phys. A 792, 264 (2007)

61. D. Amati, L. Caneschi, R. Jengo, Nucl. Phys. B 101, 397 (1975)

62. R. Jengo, Nucl. Phys. B 108, 447 (1976)

63. M. Ciafaloni, Nucl. Phys. B 146, 427 (1978)

64. S. Bondarenko, E. Gotsman, E. Levin, U. Maor, Nucl. Phys. A 683, 649 (2001)

65. E. Levin, A. Prygarin, Eur. Phys. J. C 53, 385 (2008)

66. N. Armesto, S. Bondarenko, J.G. Milhano, P. Quiroga, JHEP 0805, 103 (2008)

67. S. Bondarenko, Eur. Phys. J. C 71, 1587 (2011)

68. S. Bondarenko, L. Horwitz, J. Levitan, A. Yahalom, Nucl. Phys. A 912, 49 (2013)

69. L. Lukaszuk, B. Nicolescu, Nuovo Cimento Lett. 8, 405 (1973)

70. L.N. Lipatov, Phys. Lett. B 251, 284 (1990)

71. P. Gauron, L. Lipatov, B. Nicolescu, Phys. Lett. B 304, 334 (1993)

72. P. Gauron, L.N. Lipatov, B. Nicolescu, Z. Phys. C 63, 253 (1994)

73. L.N. Lipatov, Nucl. Phys. B 548, 328 (1999)

74. R.A. Janik, J. Wosiek, Phys. Rev. Lett. 82, 1092 (1999)

75. M.A. Braun, P. Gauron, B. Nicolescu, Nucl. Phys. B 542, 329 (1999)

76. J. Bartels, L.N. Lipatov, G.P. Vacca, Phys. Lett. B 477, 178 (2000)

77. L.N. Lipatov, A.I.P. Conf. Proc. 1523, 247 (2012)

78. H.J. de Vega, L.N. Lipatov, Phys. Lett. B 578, 335 (2004)

79. L.N. Lipatov, J. Phys. A 42, 304020 (2009)

80. J. Bartels, L.N. Lipatov, A. Prygarin, J. Phys. A 44, 454013 (2011) 